\title{
WEIGHTED THERMODYNAMIC FORMALISM ON SUBSHIFTS AND APPLICATIONS*
}

\author{
JULIEN BARRAL ${ }^{\dagger}$ AND DE-JUN FENG B $^{\ddagger}$
}

\begin{abstract}
We examine the interplay between the thermodynamic formalism and the multifractal formalism on the so-called self-affine symbolic spaces, under the specification property assumption. We investigate the properties of a weighted variational principle to derive a new result concerning the approximation of any invariant probability measure $\mu$ by sequences of weighted equilibrium states whose weighted entropies converge to the weighted entropy of $\mu$. This is a key property in the estimation of the Hausdorff dimension of sets of generic points, and then in the multifractal analysis of non homogeneous Birkhoff averages.
\end{abstract}

Key words. Thermodynamic formalism, equilibrium states, symbolic dynamics, affine invariant sets, multifractal analysis, Hausdorff dimension.

AMS subject classifications. Primary 37D35; Secondary 37B10, 37A35, 28 A78.

1. Introduction. The interplay between the thermodynamic formalism and the multifractal formalism has been rigorously examined in the literature for expanding conformal dynamical systems (see, e.g. $[15,40,38,6]$ ). In this paper, we study this relationship on the so-called self-affine symbolic spaces defined as follows.

Let $k \geq 2$. Assume that $\left(X_{i}, T_{i}\right)(i=1, \ldots, k)$ are subshifts over finite alphabets $\mathcal{A}_{i}$ such that $X_{i+1}$ is a factor of $X_{i}$ with a one-block factor map $\pi_{i}: X_{i} \rightarrow X_{i+1}$ for $i=1, \ldots, k-1$ (see Section 2 for the definitions). For convenience, we use $\pi_{0}$ to denote the identity map on $X_{1}$. Define $\tau_{i}: X_{1} \rightarrow X_{i+1}$ by $\tau_{i}=\pi_{i} \circ \pi_{i-1} \circ \cdots \circ \pi_{0}$ for $i=0,1, \ldots, k-1$.

Let $\mathbf{a}=\left(a_{1}, \ldots, a_{k}\right) \in \mathbb{R}^{k}$ so that $a_{1}>0$ and $a_{i} \geq 0$ for $i>1$. Define an ultrametric distance $d_{\mathbf{a}}$ on $X_{1}$ by

$$
d_{\mathbf{a}}(x, y)=\max \left(e^{-\frac{\left|\tau_{i-1}(x) \wedge \tau_{i-1}(y)\right|}{a_{1}+\cdots+a_{i}}}: 1 \leq i \leq k\right)
$$

where

$$
|u \wedge v|= \begin{cases}0, & \text { if } u_{1} \neq v_{1} \\ \max \left\{n: u_{j}=v_{j} \text { for } 1 \leq j \leq n\right\} & \text { if } u_{1}=v_{1}\end{cases}
$$

for $u=\left(u_{j}\right)_{j=1}^{\infty}, v=\left(v_{j}\right)_{j=1}^{\infty} \in X_{i}$. The metric space $\left(X_{1}, d_{\mathbf{a}}\right)$ is called a self-affine symbolic space. It is a natural model used to characterize the geometry of compact invariant sets on the $k$-torus under a diagonal endomorphism [7, 35, 28].

For $1 \leq i \leq k$, let $\mathcal{M}\left(X_{i}, T_{i}\right)$ denote the set of all $T_{i}$-invariant Borel probability measures on $X_{i}$, endowed with the weak-star topology. Let $\mathcal{E}\left(X_{i}, T_{i}\right)$ denote the set of ergodic measures in $\mathcal{M}\left(X_{i}, T_{i}\right)$. For $\mu \in \mathcal{M}\left(X_{1}, T_{1}\right)$, define

$$
h_{\mu}^{\mathbf{a}}\left(T_{1}\right)=\sum_{i=1}^{k} a_{i} h_{\mu \circ \tau_{i-1}^{-1}}\left(T_{i}\right),
$$

\footnotetext{
*Received December 9, 2010; accepted for publication August 2, 2011.

†LAGA (UMR 7539), Département de Mathématiques, Institut Galilée, Université Paris 13, 99 avenue Jean-Baptiste Clément, 93430 Villetaneuse, France (barral@math.univ-paris13.fr).

$\ddagger$ Department of Mathematics, The Chinese University of Hong Kong, Shatin, Hong Kong (djfeng@math.cuhk.edu.hk).
} 
which is called the a-weighted entropy of $\mu$. It was proved by Kenyon and Peres [28] that

$$
\operatorname{dim}_{H} \mu=h_{\mu}^{\mathbf{a}}\left(T_{1}\right)
$$

for each $\mu \in \mathcal{E}\left(X_{1}, T_{1}\right)$, here $\operatorname{dim}_{H} \mu$ denotes the Hausdorff dimension of $\mu$ in the metric space $\left(X_{1}, d_{\mathbf{a}}\right)$, that is, $\operatorname{dim}_{H} \mu=\inf \left\{\operatorname{dim}_{H} E\right.$ : Borel $E \subset X_{1}$ with $\left.\mu\left(X_{1} \backslash E\right)=0\right\}$.

For $\mu \in \mathcal{M}\left(X_{1}, T_{1}\right)$, the set of generic points of $\mu$ is defined by

$$
G_{\mu}\left(X_{1}, T_{1}\right)=\left\{x \in X_{1}: \lim _{n \rightarrow \infty} \frac{1}{n} \sum_{j=0}^{n-1} \phi\left(T_{1}^{j} x\right)=\int \phi d \mu \text { for all } \phi \in C\left(X_{1}\right)\right\}
$$

where $C\left(X_{1}\right)$ denotes the set of all real continuous functions on $X_{1}$.

We are going to establish a connection between the thermodynamic formalism and the multifractal formalism on $\left(X_{1}, d_{\mathbf{a}}\right)$ by considering the Hausdorff dimension of $G_{\mu}\left(X_{1}, T_{1}\right)$ and the multifractal analysis of (non-homogeneous) Birkhoff averages (which will be defined a little bit later) of continuous functions in $\left(X_{1}, d_{\mathbf{a}}\right)$.

A key notion introduced for the above study is the weighted topological pressure, defined for each $\phi \in C\left(X_{1}\right)$ by

$$
P^{\mathbf{a}}\left(T_{1}, \phi\right)=\sup \left\{\int \phi d \mu+h_{\mu}^{\mathbf{a}}\left(T_{1}\right): \mu \in \mathcal{M}\left(X_{1}, T_{1}\right)\right\} .
$$

Clearly the supremum is attainable, since the weighted entropy $h_{(\cdot)}^{\mathbf{a}}\left(T_{1}\right)$ is upper semicontinuous on $\mathcal{M}\left(X_{1}, T_{1}\right)$. Each measure $\mu$ which attains the supremum is called an $\mathbf{a}$-weighted equilibrium state of $\phi$. When $\mathbf{a}=(1,0, \ldots, 0)$, the a-weighted topological pressure and a-weighted equilibrium states are reduced back to the classical topological pressure and equilibrium states (cf. [43, 44, 38]).

We say that the subshift $X_{1}$ satisfies specification if there exists $s \in \mathbb{N}$ such that, for any two words $I$ and $J$ that are legal in $X_{1}$, there is a word $K$ of length $s$ such that the word $I K J$ is legal in $X_{1}$. For more details about this definition, see Section 2.

For $n \in \mathbb{N}$, let $\mathcal{L}_{n}\left(X_{1}\right)$ denote the $n$-th language of $X_{1}$ (see Section 2.1). Furthermore, for $I \in \mathcal{L}_{n}\left(X_{1}\right)$, let $[I]$ denote the $n$-th cylinder in $X_{1}$ associated with $I$ (see Section 2.1). Define

$$
\Omega_{n}=\left\{(\eta([I]))_{I \in \mathcal{L}_{n}\left(X_{1}\right)}: \eta \in \mathcal{M}\left(X_{1}, T_{1}\right)\right\} .
$$

Clearly $\Omega_{n}$ is a convex set. Let $\operatorname{ri}\left(\Omega_{n}\right)$ denote the relative interior of $\Omega_{n}$. Say that $f \in C\left(X_{1}\right)$ is an $n$-symbol function if $f(x)$ only depends on the first $n$-coordinates of $x$.

One of the main results of this paper is the following.

THEOREM 1.1. Assume that $\left(X_{1}, T_{1}\right)$ is a subshift satisfying specification.

(i) Let $n \in \mathbb{N}$ and $\eta \in \mathcal{M}\left(X_{1}, T_{1}\right)$. If $(\eta([I]))_{I \in \mathcal{L}_{n}\left(X_{1}\right)} \in$ ri( $\left(\Omega_{n}\right)$, then there is a unique measure $\mu=\mu(\mathbf{a}, \eta, n)$ in $\mathcal{M}\left(X_{1}, T_{1}\right)$ attaining the following supremum

$$
\sup \left\{h_{\mu}^{\mathbf{a}}\left(T_{1}\right): \mu([I])=\eta([I]) \text { for all } I \in \mathcal{L}_{n}\left(X_{1}\right)\right\} .
$$

Furthermore, $\mu(\mathbf{a}, \eta, n)$ is the a-weighted equilibrium state of some $n$-symbol function. 
(ii) For any $\eta \in \mathcal{M}\left(X_{1}, T_{1}\right)$, there exists $\left(\mu_{n}\right)_{n=1}^{\infty} \subset \mathcal{M}\left(X_{1}, T_{1}\right)$ converging to $\eta$ in the weak-star topology such that for each $n, \mu_{n}$ is the a-weighted equilibrium state of some n-symbol function and

$$
h_{\mu_{n}}^{\mathbf{a}}\left(T_{1}\right) \geq h_{\mu}^{\mathbf{a}}\left(T_{1}\right), \quad \lim _{n \rightarrow \infty} h_{\mu_{n}}^{\mathbf{a}}\left(T_{1}\right)=h_{\mu}^{\mathbf{a}}\left(T_{1}\right) .
$$

The above result is well known in the special case that $\mathbf{a}=(1,0, \ldots, 0)$ and $X_{1}$ is an irreducible subshift of finite type, for which $\mu(\mathbf{a}, \eta, n)$ is a $(n-1)$-step Markov measure (see, e.g. [19]). We remark that in our general setting, $\mu(\mathbf{a}, \eta, n)$ is typically not a Markov measure of any order even in the full shift case. In the following, we give a simple example (see Section 4 for a proof).

EXAmPle 1.2. Let $X_{1}=\{a, b, c\}^{\mathbb{N}}$ and $X_{2}=\{1,2\}^{\mathbb{N}}$. Let $\pi: X_{1} \rightarrow X_{2}$ be a one-block factor map induced by $a, b \mapsto 1$ and $c \mapsto 2$. Let $\mathbf{a}=\left(a_{1}, a_{2}\right)$ with $a_{1}, a_{2}>0$. Let $\eta$ be a fully supported measure in $\mathcal{M}\left(X_{1}, T_{1}\right)$. If

$$
\frac{\eta([a c])}{\eta([a])}=\frac{\eta([b c])}{\eta([b])} \quad \text { or } \quad \frac{\eta([c a])}{\eta([a])}=\frac{\eta([c b])}{\eta([b])},
$$

then $\mu(\mathbf{a}, \eta, 2)$ is a one-step Markov measure; otherwise $\mu(\mathbf{a}, \eta, 2)$ is not a Markov measure of any order.

We point out that when $X_{1}=\mathcal{A}_{1}^{\mathbb{N}}$ is a full shift and $f$ is a continuous function on $X_{1}$ with sufficiently regularity (Hölder continuity, for instance), the a-weighted equilibrium state of $f$ is quasi-Bernoulli (see Theorem 3.5), furthermore it is the classical equilibrium state of some continuous function $g$ on $X_{1}$ (see Remark 3.7). Recall that a probability measure $\mu$ on $\mathcal{A}_{1}^{\mathbb{N}}$ is called quasi-Bernoulli if there exists $C>1$ such that

$$
\frac{1}{C} \mu([I]) \mu([J]) \leq \mu([I J]) \leq C \mu([I]) \mu([J]), \quad \forall I, J \in \mathcal{A}_{1}^{*}=\bigcup_{n=0}^{\infty} \mathcal{A}_{1}^{n} .
$$

Theorem 1.1 might have its own interest in ergodic theory. It is crucial in our study of the Hausdorff dimension of generic points of invariant measures. Indeed, we have

THEOREM 1.3. Assume that $\left(X_{1}, T_{1}\right)$ is a subshift satisfying specification. Then for any $\mu \in \mathcal{M}\left(X_{1}, T_{1}\right)$,

$$
\operatorname{dim}_{H} G_{\mu}\left(X_{1}, T_{1}\right)=h_{\mu}^{\mathbf{a}}\left(T_{1}\right) .
$$

We remark that $G_{\mu}\left(X_{1}, T_{1}\right) \neq \emptyset$ for each $\mu \in \mathcal{M}\left(X_{1}, T_{1}\right)([39,20])$. Theorem 1.3 is only known in the literature for the case that $\mathbf{a}=(1,0, \ldots, 0)$ (cf. $[9,11$, $39,20])$, which corresponds to the conformal case. Our proof of the lower bound $\operatorname{dim}_{H} G_{\mu}\left(X_{1}, T_{1}\right) \geq h_{\mu}^{\mathbf{a}}\left(T_{1}\right)$ in the general case is based on a delicate concatenation of quasi-Bernoulli measures provided by Theorem 1.1. The upper bound is not a simple adaptation of McMullen argument; we need to overcome a difficulty coming from the fact that, in the specification case, weighted equilibrium states of Hölder continuous functions might not be quasi-Bernoulli. It is worth to point out that (1.6) still holds for any $\mu \in \mathcal{E}\left(X_{1}, T_{1}\right)$ without assuming the specification property of $X_{1}$ (see Remark $1.5)$; this fact was proved by Bowen [9] in the case that $\mathbf{a}=(1,0, \ldots, 0)$. 
To formulate our result on the multifractal analysis, let $d \in \mathbb{N}$ and let $\boldsymbol{\Phi}_{j}, j=$ $1, \ldots, r$, be $\mathbb{R}^{d}$-valued continuous functions on $X_{1}$. Let $\mathbf{c}=\left(c_{1}, \ldots, c_{r}\right) \in \mathbb{R}^{r}$ with $c_{i}>0$ for all $i$. For $\alpha=\left(\alpha_{1}, \ldots, \alpha_{d}\right) \in \mathbb{R}^{d}$, define the level set

$$
E_{\left\{\boldsymbol{\Phi}_{j}\right\}_{j=1}^{r}, \mathbf{c}}(\alpha)=\left\{x \in X_{1}: \lim _{n \rightarrow \infty} \sum_{j=1}^{r} \frac{S_{\left\lfloor c_{j} n\right\rfloor} \boldsymbol{\Phi}_{j}(x)}{\left\lfloor c_{j} n\right\rfloor}=\alpha\right\},
$$

where $S_{n} \boldsymbol{\Phi}_{j}(x):=\sum_{\ell=0}^{n-1} \boldsymbol{\Phi}_{j}\left(T_{1}^{\ell} x\right)$, and $\lfloor x\rfloor$ denotes the largest integer not greater

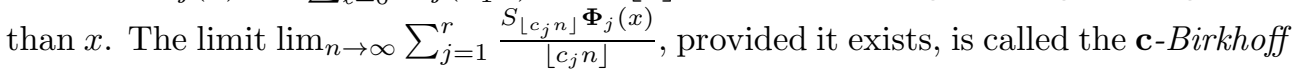
average of $\left(\boldsymbol{\Phi}_{1}, \ldots, \boldsymbol{\Phi}_{r}\right)$ at $x$. When $\mathbf{c}$ is a multiple of $(1, \ldots, 1)$, this average reduces back to the Birkhoff average of $\sum_{j=1}^{r} \boldsymbol{\Phi}_{j}$.

THEOREM 1.4. Assume that the subshift $X_{1}$ satisfies specification. Let $\mathbf{\Phi}=$ $\sum_{j=1}^{r} \boldsymbol{\Phi}_{j}$. Set $L_{\boldsymbol{\Phi}}=\left\{\int \boldsymbol{\Phi} d \mu: \mu \in \mathcal{M}\left(X_{1}, T_{1}\right)\right\}$.

1. For $\alpha \in \mathbb{R}^{d}, E_{\left\{\boldsymbol{\Phi}_{j}\right\}_{j=1}^{r}, \mathbf{c}}(\alpha) \neq \emptyset$ if and only if $\alpha \in L_{\boldsymbol{\Phi}}$.

2. For $\alpha \in L_{\boldsymbol{\Phi}}$, we have

$$
\begin{aligned}
\operatorname{dim}_{H} E_{\left\{\boldsymbol{\Phi}_{j}\right\}_{j=1}^{r}, \mathbf{c}}(\alpha) & =\max \left\{h_{\mu}^{\mathbf{a}}\left(T_{1}\right): \mu \in \mathcal{M}\left(X_{1}, T_{1}\right), \boldsymbol{\Phi}_{*}(\mu)=\alpha\right\} \\
& =\inf \left\{P^{\mathbf{a}}\left(T_{1}, \mathbf{q} \cdot \mathbf{\Phi}\right)-\alpha \cdot \mathbf{q}: \mathbf{q} \in \mathbb{R}^{d}\right\}
\end{aligned}
$$

where $\mathbf{a} \cdot \mathbf{b}$ denotes the standard dot product of $\mathbf{a}, \mathbf{b} \in \mathbb{R}^{d}$.

3. Suppose that $L_{\boldsymbol{\Phi}}$ is not a singleton. Then the set $X_{1} \backslash \bigcup_{\alpha \in L_{\boldsymbol{\Phi}}} E_{\left\{\boldsymbol{\Phi}^{(j)}\right\}, \mathbf{c}}(\alpha)$ has the same Hausdorff dimension as $X_{1}$.

Theorem 1.4 is concerned with the multifractal analysis of the level set of nonhomogeneous Birkoff averages, which was initially motivated by the study of the multifractal analysis of a-weighted Gibbs measures (see Section 6). It also provides a unified way to study the multifractal analysis of Birkhoff averages and Gibbs measures. As far as we know, this result is new when $r \geq 2$, even in the case $\mathbf{a}=(1,0, \ldots, 0)$. The level sets $E_{\left\{\boldsymbol{\Phi}_{j}\right\}_{j=1}^{r}, \mathbf{c}}(\alpha)$ do depend on $\mathbf{c}$ (see Example 6.2). In the literature, there are some works considering the multifractal analysis of Birkhoff averages of Hölder continuous functions, and Gibbs measures on self-affine sponges [31, 37, 2, 3]. However no relation between the Hausdorff spectra and any dynamical quantity like entropy was found in these papers. Moreover, the methods employed in these papers do not provide the whole Hausdorff spectrum, and they can not be used to study the cases of general continuous functions and weak Gibbs measures.

REMARK 1.5. Our proof of the upper bound for the Hausdorff dimension of level sets of non homogeneous Birkhoff averages does not use the specification property; it follows that the upper bound $\operatorname{dim}_{H} G_{\mu}\left(X_{1}, T_{1}\right) \leq h_{\mu}^{\mathbf{a}}\left(T_{1}\right)$ holds for any $\mu \in \mathcal{M}\left(X_{1}, T_{1}\right)$ without this property. Consequently, due to (1.2), (1.6) holds for any $\mu \in \mathcal{E}\left(X_{1}, T_{1}\right)$ without assuming the specification property of $X_{1}$. Furthermore, the upper bound

$$
\operatorname{dim}_{P} G_{\mu}\left(X_{1}, T_{1}\right) \leq h_{\mu}^{\mathbf{a}}\left(T_{1}\right)
$$

also holds for any $\mu \in \mathcal{M}\left(X_{1}, T_{1}\right)$ in the general subshift setting, where $\operatorname{dim}_{P}$ denotes the packing dimension (see Remark 5.10 for details). Hence we have $\operatorname{dim}_{P} G_{\mu}\left(X_{1}, T_{1}\right)=h_{\mu}^{\mathbf{a}}\left(T_{1}\right)$ in the setting of Theorem 1.3.

The paper is organized as follows. Some definitions and known results on subadditive thermodynamic formalism on subshifts are given in Section 2. In Section 3, 
we study the weighted topological pressure and equilibrium states for sub-additive potentials. In Section 4, we prove Theorem 1.1 and the statement given in Example 1.2. Then, in Section 5 we prove Theorems 1.3-1.4. In Section 6, we give some extensions of the multifractal analysis to asymptotically additive potentials and weighted equilibrium states. In Appendix A, we summarize the main notation and conventions used in this paper.

2. Sub-additive thermodynamic formalism on subshifts. In this section, we present some definitions and known results about the sub-additive thermodynamic formalism on subshifts, which plays an important role in our study of weighted topological pressures and weighted equilibrium states.

2.1. Subshifts over finite alphabets. Let $p \geq 2$ be an integer and $\mathcal{A}=$ $\{1, \ldots, p\}$. Denote

$$
\mathcal{A}^{\mathbb{N}}=\left\{\left(x_{i}\right)_{i=1}^{\infty}: x_{i} \in \mathcal{A} \text { for } i \geq 1\right\} .
$$

Then $\mathcal{A}^{\mathbb{N}}$ is compact endowed with the product discrete topology (cf. [33]). We say that $(X, T)$ is a subshift over $\mathcal{A}$, if $X$ is a compact subset of $\mathcal{A}^{\mathbb{N}}$ and $T(X) \subseteq X$, where $T$ is the left shift map on $\mathcal{A}^{\mathbb{N}}$ defined as

$$
T\left(\left(x_{i}\right)_{i=1}^{\infty}\right)=\left(x_{i+1}\right)_{i=1}^{\infty}, \quad \forall\left(x_{i}\right)_{i=1}^{\infty} \in \mathcal{A}^{\mathbb{N}} .
$$

In particular, $(X, T)$ is called the full shift over $\mathcal{A}$ if $X=\mathcal{A}^{\mathbb{N}}$. For any $n \in \mathbb{N}$ and $I \in \mathcal{A}^{n}$, we write

$$
[I]=\left\{\left(x_{i}\right)_{i=1}^{\infty} \in \mathcal{A}^{\mathbb{N}}: x_{1} \ldots x_{n}=I\right\}
$$

and call it an $n$-th cylinder in $\mathcal{A}^{\mathbb{N}}$.

The language $\mathcal{L}(X)$ of a subshift $X$ is the set of all finite words (including the empty word $\varepsilon$ ) that occur as consecutive strings $x_{1} \ldots x_{n}$ in the sequences $x=\left(x_{i}\right)_{i=1}^{\infty}$ which comprise $X$. That is,

$$
\mathcal{L}(X)=\left\{I \in \mathcal{A}^{*}: I=x_{1} \ldots x_{n} \text { for some } x=\left(x_{i}\right)_{i=1}^{\infty} \in X \text { and } n \geq 1\right\} \cup\{\varepsilon\} .
$$

Denote by $|I|$ the length of a word $I$. For $n \geq 0$, denote

$$
\mathcal{L}_{n}(X)=\{I \in \mathcal{L}(X):|I|=n\},
$$

and we call $\mathcal{L}_{n}(X)$ the $n$-th language of $X$.

Let $s \in \mathbb{N}$. A subshift $X$ is said to satisfy s-specification if for any $I, J \in \mathcal{L}(X)$, there exists $K \in \mathcal{L}_{s}(X)$ such that $I K J \in \mathcal{L}(X)$. We say that $X$ satisfies specification if it satisfies $s$-specification for some $s \in \mathbb{N}$.

Let $(X, T)$ and $(Y, S)$ be two subshifts over finite alphabets $\mathcal{A}$ and $\mathcal{D}$, respectively. We say that $Y$ is a factor of $X$, if there is a continuous surjective map $\pi: X \rightarrow Y$ such that $\pi T=S \pi$. Here $\pi$ is called a factor map. Furthermore $\pi$ is called a one-block factor map if there exists a map $\pi: \mathcal{A} \rightarrow \mathcal{D}$ such that

$$
\pi\left(\left(x_{i}\right)_{i=1}^{\infty}\right)=\left(\pi\left(x_{i}\right)\right)_{i=1}^{\infty}, \quad \forall\left(x_{i}\right)_{i=1}^{\infty} \in X .
$$

It is well known (see, e.g. [33, Proposition 1.5.12]) that each factor map $\pi: X \rightarrow Y$ between two subshifts $X$ and $Y$, will become a one-block factor map if we enlarge the alphabet for $X$ and recode $X$ appropriately. 
2.2. Sub-additive thermodynamic formalism. Let $(X, T)$ be a subshift over a finite alphabet $\mathcal{A}$. Let $\Phi=\left(\log \phi_{n}\right)_{n=1}^{\infty}$ be a sequence of real functions on $X$. We say that $\Phi$ is a sub-additive potential and write $\Phi \in \mathcal{C}_{s}(X, T)$ if $\phi_{n}$ is non-negative and upper semi-continuous ${ }^{1}$ on $X$ for each $n$ and there exists a constant $c>0$ such that

$$
\phi_{n+m}(x) \leq c \phi_{n}(x) \phi_{m}\left(T^{n} x\right), \quad \forall x \in X, n, m \in \mathbb{N} .
$$

(we admit that $\phi_{n}$ takes the value zero). More generally, $\Phi=\left(\log \phi_{n}\right)_{n=1}^{\infty}$ is said to be an asymptotically sub-additive potential and write $\Phi \in \mathcal{C}_{a s s}(X, T)$ if for any $\varepsilon>0$, there exists a sub-additive potential $\Psi=\left(\log \psi_{n}\right)_{n=1}^{\infty}$ on $X$ such that

$$
\limsup _{n \rightarrow \infty} \frac{1}{n} \sup _{x \in X}\left|\log \phi_{n}(x)-\log \psi_{n}(x)\right| \leq \varepsilon,
$$

where we take the convention $\log 0-\log 0=0$. Furthermore $\Phi$ is called an asymptotically additive potential and write $\Phi \in \mathcal{C}_{a s a}(X, T)$ if both $\Phi$ and $-\Phi$ are asymptotically sub-additive, where $-\Phi$ denotes $\left(\log \left(1 / \phi_{n}\right)\right)_{n=1}^{\infty}$. We say that $\Phi$ is almost additive if $\phi_{n}$ is positive and continuous on $X$ for each $n$ and there is a constant $c>0$ such that

$$
\frac{1}{c} \phi_{n}(x) \phi_{m}\left(T^{n} x\right) \leq \phi_{n+m}(x) \leq c \phi_{n}(x) \phi_{m}\left(T^{n} x\right), \quad \forall x \in X, n, m \in \mathbb{N} .
$$

For convenience, we denote by $\mathcal{C}_{a a}(X, T)$ the collection of almost additive potentials on $X$. In particular, $\Phi$ is called additive if each $\phi_{n}$ is a continuous positive-valued function so that $\phi_{n+m}(x)=\phi_{n}(x) \phi_{m}\left(T^{n} x\right)$ for all $x \in X$ and $m, n \in \mathbb{N}$; in this case, there is a continuous real function $g$ on $X$ such that $\phi_{n}(x)=\exp \left(\sum_{i=0}^{n-1} g\left(T^{i} x\right)\right)$ for each $n$.

For $\Phi=\left(\log \phi_{n}\right)_{n=1}^{\infty} \in \mathcal{C}_{a s s}(X, T)$, and a compact set $K \subseteq X$, define

$$
P_{n}(T, \Phi, K)=\sum_{I \in \mathcal{A}^{n},[I] \cap K \neq \emptyset} \sup _{x \in[I] \cap K} \phi_{n}(x) .
$$

and

$$
P(T, \Phi, K)=\limsup _{n \rightarrow \infty} \frac{1}{n} \log P_{n}(T, \Phi, K) .
$$

For $\mu \in \mathcal{M}(X, T)$, let $h_{\mu}(T)$ denote the measure-theoretic entropy of $\mu$ with respect to $T$, and write

$$
\Phi_{*}(\mu)=\lim _{n \rightarrow \infty} \frac{1}{n} \int_{X} \log \phi_{n}(x) d \mu(x) .
$$

The existence of the limit (which may take value $-\infty$ ) in $(2.3)$ follows from the subadditivity of $\Phi$. We list below some basic properties of asymptotically sub-additive potentials.

Lemma 2.1 ([23], Appendix A). Let $\Phi=\left(\log \phi_{n}\right)_{n=1}^{\infty} \in \mathcal{C}_{a s s}(X, T)$. Then we have the following properties.

(i) Let $\mu \in \mathcal{M}(X, T)$. The limit $\lambda_{\Phi}(x):=\lim _{n \rightarrow \infty} \frac{1}{n} \log \phi_{n}(x)$ exists (which may take value $-\infty)$ for $\mu$-a.e. $x \in X$, and $\int \lambda_{\Phi}(x) d \mu(x)=\Phi_{*}(\mu)$. When $\mu$ is ergodic, $\lambda_{\Phi}(x)=\Phi_{*}(\mu)$ for $\mu$-a.e. $x \in X$.

\footnotetext{
${ }^{1}$ In the previous definition in $[12,23], \phi_{n}$ is assumed to be non-negative and continuous.
} 
(ii) The map $\Phi_{*}: \mathcal{M}(X, T) \rightarrow \mathbb{R} \cup\{-\infty\}$ is upper semi-continuous, and there is $C \in \mathbb{R}$ such that for all $\mu \in \mathcal{M}(X, T), \lambda_{\Phi}(x) \leq C \mu$-a.e and $\Phi_{*}(\mu) \leq C$. If $\Phi \in \mathcal{C}_{\text {asa }}(X, T), \Phi_{*}$ is continuous on $\mathcal{M}(X, T)$.

(iii) $\Phi \in \mathcal{C}_{\text {asa }}(X, T)$ if and only if for any $\varepsilon>0$, there exists a continuous function $g$ on $X$ such that

$$
\limsup _{n \rightarrow \infty} \frac{1}{n} \sup _{x \in X}\left|\log \phi_{n}(x)-S_{n} g(x)\right| \leq \varepsilon,
$$

where $S_{n} g(x):=\sum_{j=0}^{n-1} g\left(T^{j} x\right)$.

The following variational principle plays a key role in our analysis.

Proposition $2.2([12])$. Let $P(T, \Phi, X)$ be defined as in (2.2). Then for any $\Phi \in \mathcal{C}_{\text {ass }}(X, T)$, we have the following variational principle:

$$
P(T, \Phi, X)=\sup \left\{\Phi_{*}(\mu)+h_{\mu}(T): \mu \in \mathcal{M}(X, T)\right\} .
$$

We call $P(T, \Phi):=P(T, \Phi, X)$ the topological pressure of $\Phi$. We remark that the variational principle for sub-additive potentials has been studied in $[17,4,21,27$, $30,5,36]$ under additional assumptions on the corresponding sub-additive potential and TDS. Say that $\mu \in \mathcal{M}(X, T)$ is an equilibrium state of $\Phi$ if the supremum in (2.4) is attained at $\mu$. Note that $\Phi_{*}(\cdot)$ is upper semi-continuous on $\mathcal{M}(X, T)$ (cf. Lemma 2.1(ii)), and so is $h_{(\cdot)}(T)$ for subshifts. Hence any $\Phi \in \mathcal{C}_{\text {ass }}(X, T)$ has at least one equilibrium state.

Proposition $2.3([5,36])$. Let $(X, T)$ be a full shift or mixing subshift of finite type. Let $\Phi=\left(\log \phi_{n}\right)_{n=1}^{\infty} \in \mathcal{C}_{a a}(X, T)$. Assume that $\Phi$ has the bounded distortion property. Then $\Phi$ has a unique equilibrium state $\mu$. Furthermore, there exists a constant $c>0$ such that for any $n \in \mathbb{N}$ and $x=\left(x_{i}\right)_{i=1}^{\infty} \in X$,

$$
c^{-1} \leq \frac{\mu\left(\left[x_{1} \ldots x_{n}\right]\right)}{\exp (-n P(T, \Phi)) \phi_{n}(x)} \leq c .
$$

REMARK 2.4 .

(1) Lemma 2.1 was proved in [23] under a slightly stronger assumption that $\phi_{n}$ is continuous on $X$. However it is easy to extend the result to the case that $\phi_{n}$ is upper semi-continuous, by using the property that, if $f$ is an upper semi-continuous function on $X$, then so is the map $\mu \mapsto \int f d \mu$ on $\mathcal{M}(X)$ (cf. [16, (A8)]). Similarly, Proposition 2.2 was only stated in [12] for subadditive potentials under a slight stronger assumption that $\phi_{n}$ is continuous. However, the proof given there works well for this new setting. Indeed, using the property we mentioned above, one sees that Lemma 2.3 in [12] can be extended to the case $\Phi \in \mathcal{C}_{a s s}(X, T)$.

(2) A special case of Proposition 2.3 was first proved in [24, 21] for the almost additive potentials given by

$$
\phi_{n}(x)=\left\|M(x) M(T x) \ldots M\left(T^{n-1} x\right)\right\|, \quad n \in \mathbb{N},
$$

where $M$ is a Hölder continuous function taking values in the set of $d \times d$ positive matrices. 
(3) According to Lemma 2.1(iii), for $\mu \in \mathcal{M}(X, T)$, the set $G_{\mu}\left(X, T_{1}\right)$ of generic points of $\mu$ defined as in (1.3) is just equal to

$$
\left\{x \in X: \lim _{n \rightarrow \infty} \frac{\log \phi_{n}(x)}{n}=\Phi_{*}(\mu), \quad \forall \Phi=\left(\log \phi_{n}\right)_{n=1}^{\infty} \in \mathcal{C}_{\text {asa }}(X, T)\right\} .
$$

2.3. Relativized sub-additive thermodynamic formalism. Let $\pi: X \rightarrow$ $Y$ be a one-block factor map between two subshifts $(X, T)$ and $(Y, S)$. The following relativized variational principle was proved in [45] for sub-additive potentials $\Phi=$ $\left.\left(\log \phi_{n}\right\}\right)_{n=1}^{\infty}$ with $\phi_{n}$ being continuous, under a general random setting. It does hold for $\Phi \in \mathcal{C}_{\text {ass }}(X, T)$ by modifying the proof in [45] slightly. This extends the relativized variational principle of Ledrappier and Walters [32] for additive potentials.

Proposition 2.5. Let $\Phi \in \mathcal{C}_{a s s}(X, T)$ and $\nu \in \mathcal{M}(Y, S)$. Then

$$
\sup \left\{\Phi_{*}(\mu)+h_{\mu}(T)-h_{\nu}(S)\right\}=\int_{Y} P\left(T, \Phi, \pi^{-1}(y)\right) d \nu(y),
$$

where the supremum is taken over the set of $\mu \in \mathcal{M}(X, T)$ such that $\mu \circ \pi^{-1}=\nu$, $P\left(T, \Phi, \pi^{-1}(y)\right)$ is defined as in $(2.2)$.

By the upper semi-continuity of $\Phi_{*}(\cdot)$ and $h_{(\cdot)}(T)$ on $\mathcal{M}(X, T)$, the supremum in (2.5) is attainable. Any measure $\mu \in \mathcal{M}(X, T)$ for which the supremum in (2.5) is attained at $\mu$ is called a conditional equilibrium state of $\Phi$ with respect to $\nu$.

3. Weighted thermodynamic formalism. In this section, we define the weighted topological pressure for general asymptotically sub-additive potentials, and we discuss the uniqueness and Gibbs properties of weighted equilibrium states. These properties are needed in the proofs of our main results listed in Section 1.

First we recall our basic settings. Let $k \geq 2$. Assume that $\left(X_{i}, T_{i}\right)(i=1, \ldots, k)$ are subshifts over finite alphabets $\mathcal{A}_{i}$ such that $X_{i+1}$ is a factor of $X_{i}$ with a one-block factor map $\pi_{i}: X_{i} \rightarrow X_{i+1}$ for $i=1, \ldots, k-1$. For convenience, we use $\pi_{0}$ to denote the identity map on $X_{1}$. Define $\tau_{i}: X_{1} \rightarrow X_{i+1}$ by $\tau_{i}=\pi_{i} \circ \pi_{i-1} \circ \cdots \circ \pi_{0}$ for $i=0,1, \ldots, k-1$.

Let $\mathbf{a}=\left(a_{1}, \ldots, a_{k}\right) \in \mathbb{R}^{k}$ so that $a_{1}>0$ and $a_{i} \geq 0$ for $i>1$. For $\Phi \in$ $\mathcal{C}_{a s s}\left(X_{1}, T_{1}\right)$. We define the a-weighted topological pressure of $\Phi$ as

$$
P^{\mathbf{a}}\left(T_{1}, \Phi\right)=\sup \left\{\Phi_{*}(\mu)+h_{\mu}^{\mathbf{a}}\left(T_{1}\right): \mu \in \mathcal{M}\left(X_{1}, T_{1}\right)\right\} .
$$

By Lemma 2.1(ii), $\Phi_{*}(\cdot)$ is upper semi-continuous on $\mathcal{M}\left(X_{1}, T_{1}\right)$, and so is $h_{(\cdot)}^{\mathbf{a}}\left(T_{1}\right)$, whence the above supremum is attainable. Each measure $\mu$ which attains the supremum is called an a-weighted equilibrium state of $\Phi$.

For $i=1, \ldots, k-1$, we define $\theta_{i}: \mathcal{C}_{a s s}\left(X_{i}, T_{i}\right) \rightarrow \mathcal{C}_{a s s}\left(X_{i+1}, T_{i+1}\right)$ by $\left(\log \phi_{n}\right)_{n=1}^{\infty} \mapsto\left(\log \psi_{n}\right)_{n=1}^{\infty}$, where

$$
\psi_{n}(y)=\left(\sum_{I \in \mathcal{A}_{i}^{n}:[I] \cap \pi_{i}^{-1}(y) \neq \emptyset} \sup _{x \in[I] \cap \pi_{i}^{-1}(y)} \phi_{n}(x)^{1 / A_{i}}\right)^{A_{i}}
$$

for $y \in X_{i+1}$, with $A_{i}=a_{1}+\cdots+a_{i}$. In particular, let $\mathcal{S}_{\text {ass }}$ denote the collection of asymptotically sub-additive additive (scalar) sequences $\left(\log c_{n}\right)_{n=1}^{\infty}$ (a sequence $\left(\log c_{n}\right)_{n=1}^{\infty}$, where $c_{n} \geq 0$, is called asymptotically sub-additive if, for 
any $\varepsilon>0$, there exists a sequence $\left(d_{n}\right)_{n=1}^{\infty}$, so that $0 \leq d_{n+m} \leq d_{n} d_{m}$ and $\left.\lim \sup _{n \rightarrow \infty} \frac{1}{n}\left|\log c_{n}-\log d_{n}\right|<\varepsilon\right)$. Let $\theta_{k}: \mathcal{C}_{\text {ass }}\left(X_{k}, T_{k}\right) \rightarrow \mathcal{S}_{\text {ass }}$ be defined as $\left(\log \phi_{n}\right)_{n=1}^{\infty} \mapsto\left(\log c_{n}\right)_{n=1}^{\infty}$, where

$$
c_{n}=\left(\sum_{I \in \mathcal{A}_{k}^{n}} \sup _{x \in[I]} \phi_{n}(x)^{1 / A_{k}}\right)^{A_{k}} .
$$

Our first result in this section is the following.

Theorem 3.1. Let $\Phi=\left(\log \phi_{n}\right)_{n=1}^{\infty} \in \mathcal{C}_{\text {ass }}\left(X_{1}, T_{1}\right)$. Then

(i) For any $1 \leq i \leq k-1, P^{\mathbf{a}}\left(T_{1}, \Phi\right)=P^{\left(\sum_{j=1}^{i+1} a_{j}, a_{i+2}, \ldots, a_{k}\right)}\left(T_{i+1}, \theta_{i} \circ \cdots \circ \theta_{1}(\Phi)\right)$.

(ii) $P^{\mathbf{a}}\left(T_{1}, \Phi\right)=\lim _{n \rightarrow \infty}(1 / n) \log c_{n}$, where $\left(c_{n}\right)_{n=1}^{\infty}=\theta_{k} \circ \cdots \circ \theta_{1}(\Phi)$.

(iii) $\mu \in \mathcal{M}\left(X_{1}, T_{1}\right)$ is an a-weighted equilibrium state of $\Phi$ if and only if $\mu \circ \tau_{k-1}^{-1}$ is an equilibrium state of $\frac{\theta_{k-1} \circ \cdots \circ \theta_{1}(\Phi)}{a_{1}+\cdots+a_{k}}$ and, for $i=k-2, k-3, \ldots, 0, \mu \circ \tau_{i}^{-1}$ is a conditional equilibrium state of $\frac{\theta_{i} \circ \cdots \circ \theta_{1}(\Phi)}{a_{1}+\cdots+a_{i+1}}$ with respect to $\mu \circ \tau_{i+1}^{-1}$.

The above result establishes the relation between weighted topological pressures and non-weighted ones, as well as the relation between weighted equilibrium states, non-weighted equilibrium sates and conditional equilibrium states. We remark that Theorem 3.1 was proved in [22] for a special class of sub-additive potentials $\left(\log \phi_{n}\right)_{n=1}^{\infty}$, where $\phi_{n}$ are assumed to be $n$-symbol functions. Yayama [47, 46] also considered the case $\Phi=0$ independently.

The following simple lemma plays a key role in the proof of Theorem 3.1.

Lemma 3.2. Let $\pi: X \rightarrow Y$ be a one-block factor map between two subshifs $(X, T)$ and $(Y, S)$. Let $\Phi=\left(\log \phi_{n}\right)_{n=1}^{\infty} \in \mathcal{C}_{a s s}(X, T)$ and $\nu \in \mathcal{M}(Y, S)$. Then we have

$$
\sup \left\{\Phi_{*}(\mu)+h_{\mu}(T)-h_{\nu}(S): \mu \in \mathcal{M}(X, T), \mu \circ \pi^{-1}=\nu\right\}=\Psi_{*}(\nu),
$$

where $\Psi=\left(\log \psi_{n}\right)_{n=1}^{\infty} \in \mathcal{C}_{\text {ass }}(Y, S)$ is defined by

$$
\psi_{n}(y)=\sum_{I \in \mathcal{A}^{n}:[I] \cap \pi^{-1}(y) \neq \emptyset} \sup _{x \in[I] \cap \pi^{-1}(y)} \phi_{n}(x) .
$$

Proof. By Proposition 2.5, the left-hand side of (3.1) equals $\int P\left(T, \Phi, \pi^{-1}(y)\right) d \nu(y)$. However by (2.2)-(2.1),

$$
P\left(T, \Phi, \pi^{-1}(y)\right)=\limsup _{n \rightarrow \infty} \frac{1}{n} \log P_{n}\left(T, \Phi, \pi^{-1}(y)\right)
$$

and

$$
P_{n}\left(T, \Phi, \pi^{-1}(y)\right)=\sum_{I \in \mathcal{A}^{n}:[I] \cap \pi^{-1}(y)} \sup _{x \in[I] \cap \pi^{-1}(y)} \phi_{n}(x) .
$$

Clearly $\psi_{n}(y)=P_{n}\left(T, \Phi, \pi^{-1}(y)\right)$. It is direct to check that $\Psi=\left(\log \psi_{n}\right)_{n=1}^{\infty} \in$ $\mathcal{C}_{a s s}(Y, S)$. Hence by Lemma 2.1 ,

$$
\Psi_{*}(\nu)=\int \limsup _{n \rightarrow \infty} \frac{1}{n} \log \psi_{n}(y) d \nu(y)=\int P\left(T, \Phi, \pi^{-1}(y)\right) d \nu(y) .
$$


This finishes the proof of the lemma.

REMARK 3.3. In Lemma 3.2, it seems that $\psi_{n}$ might be discontinuous when $\phi_{n}$ is continuous. However when both $X$ and $Y$ are full shifts, if $\phi_{n}$ is continuous, then so is $\psi_{n}$.

Proof of Theorem 3.1. To show (i), we only prove the case when $i=1$. The general case then just follows by a recursive argument. Write $\mathbf{b}=\left(a_{2}, a_{3}, \ldots, a_{k}\right)$ and $\mathbf{c}=\left(a_{1}+a_{2}, a_{3}, \ldots, a_{k}\right)$. Then we have

$$
\begin{aligned}
\sup & \left\{\Phi_{*}(\mu)+h_{\mu}^{\mathbf{a}}\left(T_{1}\right), \mu \in \mathcal{M}\left(X_{1}, T_{1}\right)\right\} \\
& =\sup \left\{\Phi_{*}(\mu)+a_{1} h_{\mu}\left(T_{1}\right)+h_{\nu}^{\mathbf{b}}\left(T_{2}\right): \nu \in \mathcal{M}\left(X_{2}, T_{2}\right), \mu \in \mathcal{M}\left(X_{1}, T_{1}\right), \mu \circ \pi_{1}^{-1}=\nu\right\} \\
& =\sup \left\{A(\nu)+h_{\nu}^{\mathbf{c}}\left(T_{2}\right): \nu \in \mathcal{M}\left(X_{2}, T_{2}\right)\right\},
\end{aligned}
$$

where

$$
A(\nu):=a_{1} \sup \left\{\frac{1}{a_{1}} \Phi_{*}(\mu)+h_{\mu}\left(T_{1}\right)-h_{\nu}\left(T_{2}\right): \mu \in \mathcal{M}\left(X_{1}, T_{1}\right), \mu \circ \pi_{1}^{-1}=\nu\right\} .
$$

By Lemma 3.2, we have $A(\nu)=\Psi_{*}(\nu)$, where $\Psi=\left(\log \psi_{n}\right)_{n=1}^{\infty} \in \mathcal{C}_{a s s}(Y, S)$ is defined as

$$
\psi_{n}(y)=\left(\sum_{I \in \mathcal{A}^{n}:[I] \cap \pi^{-1}(y) \neq \emptyset} \sup _{x \in[I] \cap \pi^{-1}(y)} \phi_{n}(x)^{1 / a_{1}}\right)^{a_{1}} .
$$

That is, $\Psi=\theta_{1}(\Phi)$. Hence by $(3.2)$, we have $P^{\mathbf{a}}\left(T_{1}, \Phi\right)=P^{\mathbf{c}}\left(T_{2}, \theta_{1}(\Phi)\right)$, as desired. To see (ii), note that by (i) we have

$P^{\mathbf{a}}\left(T_{1}, \Phi\right)=P^{\left(a_{1}+\cdots+a_{k}\right)}\left(T_{k}, \Gamma\right)=\sup \left\{\Gamma_{*}(\eta)+\left(a_{1}+\cdots+a_{k}\right) h_{\eta}\left(T_{k}\right), \eta \in \mathcal{M}\left(X_{k}, T_{k}\right)\right\}$,

where $\Gamma:=\theta_{k-1} \circ \cdots \circ \theta_{1}(\Phi)$. Then (ii) follows from Proposition 2.2.

By (3.2), $\mu$ is an a-weighted equilibrium state of $\Phi$ if and only if $\mu \circ \pi_{1}^{-1}$ is a $\mathbf{c}$ weighted equilibrium state of $\theta_{1}(\Phi)$ and $\mu$ is a conditional equilibrium state of $\frac{1}{a_{1}} \theta_{1}(\Phi)$ with respect to $\mu \circ \pi_{1}^{-1}$. A recursive argument then yields (iii).

In the remaining part of this section, we assume that $X_{1}$ satisfies specification. Let $\Phi=\left(\log \phi_{n}\right)_{n=1}^{\infty} \in \mathcal{C}_{a s a}\left(X_{1}, T_{1}\right)$. Define $\phi^{(0)}: \mathcal{L}\left(X_{1}\right) \rightarrow(0, \infty)$ by $\phi^{(0)}(I)=$ $\sup _{x \in[I]} \phi_{n}(x)$ for $I \in \mathcal{L}_{n}\left(X_{1}\right)$. Furthermore, define $\phi^{(i)}: \mathcal{L}\left(X_{i+1}\right) \rightarrow[0, \infty)(i=$ $1, \ldots, k-1)$ recursively by

$$
\phi^{(i)}(J)=\left(\sum_{I \in \mathcal{L}_{n}\left(X_{i}\right): \pi_{i} I=J} \phi^{(i-1)}(I)^{\frac{1}{a_{1}+\cdots+a_{i}}}\right)^{a_{1}+\cdots+a_{i}}
$$

for $n \in \mathbb{N}, J \in \mathcal{L}_{n}\left(X_{i+1}\right)$. Furthermore, define $\phi^{(k)}: \mathbb{N} \rightarrow[0, \infty)$ by

$$
\phi^{(k)}(n)=\sum_{I \in \mathcal{L}_{n}\left(X_{k}\right)} \phi^{(k-1)}(I)^{\frac{1}{a_{1}+\cdots+a_{k}}} .
$$

Then we define the a-weighted potential associated with $\Phi$ by

$$
\Phi^{\mathbf{a}}=\left(\log \phi_{n}^{\mathbf{a}}\right)_{n=1}^{\infty}, \text { where } \phi_{n}^{\mathbf{a}}(x)=\phi^{(0)}\left(x_{\mid n}\right)^{1 / A_{1}} \prod_{i=1}^{k-1} \phi^{(i)}\left(\tau_{i}\left(x_{\mid n}\right)\right)^{1 / A_{i+1}-1 / A_{i}}
$$


where $A_{i}=a_{1}+\cdots+a_{i}$, and $x_{\mid n}:=x_{1} \ldots x_{n}$ for $x=\left(x_{i}\right)_{i=1}^{\infty} \in X_{1}$. Since there exists a sequence $\left(g^{(p)}\right)_{p \geq 1}$ of Hölder potentials such that $\lim _{p \rightarrow 0} \lim \sup _{n \rightarrow \infty} \| \Phi_{n}-$ $S_{n} g^{(p)} \|_{\infty} / n=0$ (see Lemma 2.1(iii)), it is easily seen that all the potentials $\left(\log \phi^{(i)}\left(\tau_{i-1}(\cdot \mid n)\right)_{n=1}^{\infty}\right.$ and $\left(\log \phi_{n}^{\mathbf{a}}\right)_{n=1}^{\infty}$ belong to $\mathcal{C}_{a s a}(X, T)$.

We say that $\Phi=\left(\log \phi_{n}\right)_{n=1}^{\infty} \in \mathcal{C}_{a a}\left(X_{1}, T_{1}\right)$ has the bounded distortion property if there exists a constant $c>0$ such that

$\frac{1}{c} \phi_{n}(y) \leq \phi_{n}(x) \leq c \phi_{n}(y) \quad$ whenever $x, y \in X$ are in the same $n$-th cylinder.

For two families of real numbers $\left\{a_{i}\right\}_{i \in \mathcal{I}}$ and $\left\{b_{i}\right\}_{i \in \mathcal{I}}$, we write

$$
\begin{array}{ll}
a_{i} \approx b_{i} & \text { if there is } c>0 \text { such that } \frac{1}{c} b_{i} \leq a_{i} \leq c b_{i} \text { for } i \in \mathcal{I} ; \\
a_{i} \succcurlyeq b_{i} & \text { if there is } c>0 \text { such that } a_{i} \geq c b_{i} \text { for } i \in \mathcal{I} .
\end{array}
$$

The following result was proved in [22].

TheOREM 3.4 (Theorem 7.3 in [22]). Assume that $X_{1}$ satisfies specification. Suppose that $\Phi=\left(\log \phi_{n}\right)_{n=1}^{\infty} \in \mathcal{C}_{a a}\left(X_{1}, \sigma_{X_{1}}\right)$ has the bounded distortion property. Then $\Phi$ has a unique a-weighted equilibrium state $\mu$. Furthermore, $\mu$ is ergodic and has the following properties: $\mu([I]) \approx \widetilde{\phi}^{*}(I) \succcurlyeq \widetilde{\phi}(I)$ for $I \in \mathcal{L}\left(X_{1}\right)$, where $\widetilde{\phi}, \widetilde{\phi}^{*}$ : $\mathcal{L}\left(X_{1}\right) \rightarrow[0, \infty)$ are defined respectively by

$$
\widetilde{\phi}(I)=\left(\prod_{i=0}^{k-2} \frac{\phi^{(i)}\left(\tau_{i} I\right)^{\frac{1}{a_{1}+\cdots+a_{i+1}}}}{\phi^{(i+1)}\left(\tau_{i+1} I\right)^{\frac{1}{a_{1}+\cdots+a_{i+1}}}}\right) \cdot \frac{\phi^{(k-1)}\left(\tau_{k-1} I\right)^{\frac{1}{a_{1}+\cdots+a_{k}}}}{\phi^{(k)}(n)}
$$

for $I \in \mathcal{L}_{n}\left(X_{1}\right), n \in \mathbb{N}$, and

$$
\widetilde{\phi}^{*}(I)=\sup _{m, n \geq 0} \sum_{I_{1} \in \mathcal{L}_{m}\left(X_{1}\right), I_{2} \in \mathcal{L}_{n}\left(X_{1}\right): I_{1} I I_{2} \in \mathcal{L}\left(X_{1}\right)} \widetilde{\phi}\left(I_{1} I I_{2}\right), \quad I \in \mathcal{L}\left(X_{1}\right) .
$$

Applying Theorem 3.1(ii) and Theorem 3.4 to the full shift case, we obtain

TheOREM 3.5. Assume that $X_{1}$ is a full shift.

(i) Let $\Phi=\left(\log \phi_{n}\right)_{n=1}^{\infty} \in \mathcal{C}_{a s a}\left(X_{1}, T_{1}\right)$. Then

$$
P^{\mathbf{a}}\left(T_{1}, \Phi\right)=A_{k} \lim _{n \rightarrow \infty}(1 / n) \log \phi^{(k)}(n) .
$$

(ii) Suppose that $\Phi=\left(\log \phi_{n}\right)_{n=1}^{\infty} \in \mathcal{C}_{a a}\left(X_{1}, T_{1}\right)$ has the bounded distortion property. Then $\Phi$ has a unique a-weighted equilibrium state $\mu$. Furthermore, $\mu$ is fully supported and quasi-Bernoulli, and it satisfies the following Gibbs property

$$
\mu([I]) \approx \exp \left(\frac{-n P}{A_{k}}\right) \phi_{n}^{\mathbf{a}}(I), \quad I \in \mathcal{A}_{1}^{n},
$$

where $P=P^{\mathbf{a}}\left(T_{1}, \Phi\right)$, and $\phi_{n}^{\mathbf{a}}$ is defined as in (3.4). Consequently, for $i=$ $2, \ldots, k$,

$$
\mu_{i}\left(\left[\tau_{i-1} I\right]\right) \approx \exp \left(\frac{-n P}{A_{k}}\right) \phi^{(i-1)}\left(\tau_{i-1} I\right)^{1 / A_{i}} \prod_{j=i}^{k-1} \phi^{(j)}\left(\tau_{j} I\right)^{1 / A_{j+1}-1 / A_{j}}, \quad I \in \mathcal{A}_{1}^{n},
$$

where $\mu_{i}:=\mu \circ \tau_{i-1}^{-1}$. Furthermore,

$$
\phi_{n}(x) \exp (-n P) \approx \prod_{i=1}^{k} \mu_{i}\left(\left[\tau_{i-1} x_{\mid n}\right]\right)^{a_{i}} \quad \text { for } x \in X_{1}, n \geq 1 .
$$


Definition 3.6. A Borel probability measure $\mu$ (not necessarily to be invariant) on $X_{1}$ satisfying (3.5) is called an a-weighted Gibbs measure for $\Phi$.

REMARK 3.7. In the setting of Theorem 3.4, if $\Phi=\left(S_{n} f\right)_{n=1}^{\infty}$ for $f \in C\left(X_{1}\right)$ with sufficiently regularity (for instance, the Hölder continuity), then the a-weighted Gibbs measure of $\Phi$ is the classical equilibrium state of a continuous function $g \in C\left(X_{1}\right)$ with weaker regularity. To see this, we need to use an invariance of the recent result of Chazottes and Ugalde [14, Theorem 4.1], which claims that if $\phi^{(i-1)}$ is a function on $\mathcal{A}_{i}^{*}$ such that

$$
\phi^{(i-1)}\left(x_{\mid n}\right) \approx \exp \left(S_{n} u(x)\right), \quad x \in X_{i}
$$

for some function $u(x) \in C\left(X_{i}\right)$ satisfying $\operatorname{var}_{n}(u) \leq D_{1} \exp \left(-c_{1} n^{\gamma_{1}}\right)$, where $\operatorname{var}_{n}(u)=\max _{x_{\mid n}=y_{\mid n}}|u(x)-u(y)|$, and let $\phi^{(i)}$ be defined as in (3.3), then there exist $v \in C\left(X_{i+1}\right)$ and $D_{2}>D_{1}, 0<\gamma_{2}<\gamma_{1}, 0<c_{2}<c_{1}$ so that

$$
\phi^{(i)}\left(x_{\mid n}\right) \approx \exp \left(S_{n} v(x)\right), \quad x \in X_{i+1}
$$

and $\operatorname{var}_{n}(v) \leq D_{2} \exp \left(-c_{2} n^{\gamma_{2}}\right)$. Using this claim repeatedly, we see that if $f \in C\left(X_{1}\right)$ satisfies $\operatorname{var}_{n}(f) \leq \widetilde{D} \exp \left(-\widetilde{c} n^{\widetilde{\gamma}}\right)$ for some constants $\widetilde{D}, \widetilde{c}, \widetilde{\gamma}>0$, then the potential $\phi^{\mathbf{a}}$ defined as in (3.4) will satisfy

$$
\phi^{\mathbf{a}}\left(x_{\mid n}\right) \approx \exp \left(S_{n} g(x)\right), \quad x \in X_{1}
$$

for some $g \in C\left(X_{1}\right)$ satisfying $\operatorname{var}_{n}(g) \leq D \exp \left(-c n^{\gamma}\right)$ with some constants $D, c, \gamma>$ 0 . Then according to (3.5), the a-weighted equilibrium state $\mu$ of $\Phi$ is just the equilibrium state of $g$.

In the reminder of this section, we consider the question when $\Phi, \Psi \in \mathcal{C}_{a a}\left(X_{1}, T_{1}\right)$ have the same a-weighted equilibrium state.

DeFinition 3.8. We say that two almost additive potentials $\Phi=\left(\log \phi_{n}\right)_{n=1}^{\infty}$ and $\Psi=\left(\log \psi_{n}\right)_{n=1}^{\infty}$ are cohomologous if $\sup _{n}\left\|\log \phi_{n}-\log \psi_{n}\right\|_{\infty}<\infty$, where $\|f\|_{\infty}=$ $\sup _{x \in X_{1}}|f(x)|$ for $f \in C\left(X_{1}\right.$. If there exists $C \in \mathbb{R}$ such that $\log \psi_{n}=C n$, we say that $\Phi$ is cohomologous to a constant.

The following proposition is a direct consequence of Theorem 3.5.

Proposition 3.9. Suppose $\Phi, \Psi \in \mathcal{C}_{a a}\left(X_{1}, T_{1}\right)$ satisfy the bounded distortion property. Then, $\Phi$ and $\Psi$ share the same a-weighted equilibrium state if and only if $\Phi-\Psi$ is cohomologous to a constant.

4. The proof of Theorem 1.1. In this section, we always assume that $\left(X_{1}, T_{1}\right)$ is a subshift satisfying specification. To prove Theorem 1.1, we need the following result.

Proposition 4.1. Let $\Phi_{1}, \ldots, \Phi_{d} \in \mathcal{C}_{a a}\left(X_{1}, T_{1}\right)$ satisfy the bounded distortion property. Then the map $Q: \mathbb{R}^{d} \rightarrow \mathbb{R}$ defined as

$$
\left(q_{1}, \ldots, q_{d}\right) \mapsto P^{\mathbf{a}}\left(T_{1}, \sum_{i=1}^{d} q_{i} \Phi_{i}\right),
$$

is $C^{1}$ over $\mathbb{R}^{d}$ with

$$
\nabla Q\left(q_{1}, \ldots, q_{d}\right)=\left(\left(\Phi_{1}\right)_{*}\left(\mu_{\mathbf{q}}\right), \ldots,\left(\Phi_{d}\right)_{*}\left(\mu_{\mathbf{q}}\right)\right),
$$


where $\nabla Q$ denotes the gradient of $Q, \mu_{\mathbf{q}}$ is the unique a-weighted equilibrium state of $\sum_{i=1}^{d} q_{i} \Phi_{i}$.

To prove Proposition 4.1, we need the following result coming from convex analysis.

Proposition 4.2 ([23], Proposition 2.3). Let $Z$ be a compact convex subset of a topological vector space which satisfies the first axiom of countability (i.e., there is a countable base at each point) and $U \subseteq \mathbb{R}^{d}$ a non-empty open set. Suppose $f$ : $U \times Z \rightarrow \mathbb{R} \cup\{-\infty\}$ is a map satisfying the following conditions:

(i) $f(\mathbf{q}, z)$ is convex in $\mathbf{q}$;

(ii) $f(\mathbf{q}, z)$ is affine in $z$;

(iii) $f$ is upper semi-continuous over $U \times Z$;

(iv) $g(\mathbf{q}):=\sup _{z \in Z} f(\mathbf{q}, z)>-\infty$ for any $\mathbf{q} \in U$.

For each $\mathbf{q} \in U$, denote $\mathcal{I}(\mathbf{q}):=\{z \in Z: f(\mathbf{q}, z)=g(\mathbf{q})\}$. Then

$$
\partial g(\mathbf{q})=\bigcup_{z \in \mathcal{I}(\mathbf{q})} \partial f(\mathbf{q}, z)
$$

where $\partial f(\mathbf{q}, z)$ denotes the subdifferential of $f(\cdot, z)$ at $\mathbf{q}$.

Proof of Proposition 4.1. In Proposition 4.2, we let $U=\mathbb{R}^{d}, Z=\mathcal{M}\left(X_{1}, T_{1}\right)$, and define $f: U \times Z \rightarrow \mathbb{R}$ by

$$
f(\mathbf{q}, \mu)=\sum_{i=1}^{d} q_{i}\left(\Phi_{i}\right)_{*}(\mu)+h_{\mu}^{\mathbf{a}}\left(T_{1}\right), \quad \mathbf{q}=\left(q_{1}, \ldots, q_{d}\right), \mu \in \mathcal{M}\left(X_{1}, T_{1}\right) .
$$

Set $g(\mathbf{q})=\sup _{z \in Z} f(\mathbf{q}, z)=P^{\mathbf{a}}\left(T_{1}, \sum_{i=1}^{d} q_{i} \Phi_{i}\right)$. Since $\Phi_{i} \in \mathcal{C}_{a a}\left(X_{1}, T_{1}\right), \mu \mapsto$ $\left(\Phi_{i}\right)_{*}(\mu)$ is continuous on $\mathcal{M}\left(X_{1}, T_{1}\right)$ (see Lemma 2.1(ii)). Thus, $f$ and $g$ satisfy the assumptions (i)-(iv) in Proposition 4.2. Note that $\mathcal{I}(\mathbf{q})$ just corresponds to the set of all a-weighted equilibrium states of $\sum_{i=1}^{d} q_{i} \Phi_{i}$. By Theorem 3.5, $\mathcal{I}(\mathbf{q})=\left\{\mu_{\mathbf{q}}\right\}$ is a singleton for each $\mathbf{q} \in \mathbb{R}^{d}$. By Proposition $4.2, \nabla g(\mathbf{q})=\left(\left(\Phi_{1}\right)_{*}\left(\mu_{\mathbf{q}}\right), \ldots,\left(\Phi_{d}\right)_{*}\left(\mu_{\mathbf{q}}\right)\right)$. Since $g$ is convex and differentiable on $\mathbb{R}^{d}$, it is $C^{1}$ on $\mathbb{R}^{d}$ (see, e.g. [42, Corollary 25.5.1]). This finishes the proof of Proposition 4.1.

Before proving Theorem 1.1, we still need some notation and basic facts in convex analysis. Let $g: \mathbb{R}^{d} \rightarrow \mathbb{R} \cup\{+\infty\}$ be convex and not identically equal to $+\infty$. Then the function $g^{*}: \mathbb{R}^{d} \rightarrow \mathbb{R} \cup\{+\infty\}$ defined by

$$
\mathbf{s} \mapsto g^{*}(\mathbf{s}):=\sup \left\{\mathbf{s} \cdot \mathbf{x}-g(\mathbf{x}): \mathbf{x} \in \mathbb{R}^{d}\right\}
$$

is called the conjugate function of $g$, where $\mathbf{s} \cdot \mathbf{x}$ denotes the standard dot product of $\mathbf{s}$ and $\mathbf{x}$ in $\mathbb{R}^{d}$. It is known that $g^{*}$ is also convex and not identically equal to $+\infty$ (cf. $\left[26\right.$, p. 211]). Let $g^{* *}$ denote the conjugate of $g^{*}$. The following result is well known in convex analysis (cf. [42, Theorem 12.2, Corollary 26.4.1]).

Proposition 4.3.

(i) Let $g: \mathbb{R}^{d} \rightarrow \mathbb{R} \cup\{+\infty\}$ be convex and not identically equal to $+\infty$. Let $\mathbf{x} \in \mathbb{R}^{d}$. Assume that $g$ is lower semi-continuous at $\mathbf{x}$, i.e., $\liminf _{\mathbf{y} \rightarrow \mathbf{x}} g(\mathbf{y}) \geq$ $g(\mathbf{x})$. Then $g^{* *}(\mathbf{x})=g(\mathbf{x})$.

(ii) Let $g$ be real convex and differentiable on $\mathbb{R}^{d}$. Let $D=\left\{\nabla g(\mathbf{x}): \mathbf{x} \in \mathbb{R}^{d}\right\}$. Then ri $\left(\right.$ dom $\left.g^{*}\right) \subseteq D$, where dom $g^{*}:=\left\{\mathbf{x} \in \mathbb{R}^{d}: g^{*}(\mathbf{x}) \neq+\infty\right\}$, and $\operatorname{ri}(A)$ denotes the relative interior of a convex set $A$. 
Proof of Theorem 1.1. We first prove part (i) of the theorem. Fix $n \in \mathbb{N}$ and define a function $f: \Omega_{n} \rightarrow \mathbb{R}$ by

$$
f(\mathbf{p})=\sup \left\{h_{\mu}^{\mathbf{a}}\left(T_{1}\right): \mu \in \mathcal{M}\left(X_{1}, T_{1}\right):(\mu([I]))_{I \in \mathcal{L}_{n}\left(X_{1}\right)}=\mathbf{p}\right\} .
$$

Define $g: \mathbb{R}^{\mathcal{L}_{n}\left(X_{1}\right)} \rightarrow \mathbb{R} \cup\{+\infty\}$ by

$$
g(\mathbf{p})= \begin{cases}-f(\mathbf{p}) & \text { if } \mathbf{p} \in \Omega_{n} \\ +\infty & \text { otherwise }\end{cases}
$$

It is easily checked that $g$ is convex and lower semi-continuous on $\mathbb{R}^{\mathcal{L}_{n}\left(X_{1}\right)}$. Let $g^{*}$ denote the conjugate of $g$, and $g^{* *}$ the conjugate of $g^{*}$. By Proposition 4.3(i), $g^{* *}=g$ on $\mathbb{R}^{\mathcal{L}_{n}\left(X_{1}\right)}$.

However, by the definition of $f$, we have for $\mathbf{q}=(q(I))_{I \in \mathcal{L}_{n}\left(X_{1}\right)}$,

$$
\begin{aligned}
g^{*}(\mathbf{q}) & =\sup \left\{f(\mathbf{p})+\mathbf{p} \cdot \mathbf{q}: \mathbf{p} \in \mathbb{R}^{\mathcal{L}_{n}\left(X_{1}\right)}\right\}=\sup \left\{f(\mathbf{p})+\mathbf{p} \cdot \mathbf{q}: \mathbf{p} \in \Omega_{n}\right\} \\
& =\sup \left\{\left(\sum_{I \in \mathcal{L}_{n}\left(X_{1}\right)} q(I) \int \chi_{[I]} d \mu\right)+h_{\mu}^{\mathbf{a}}\left(T_{1}\right): \mu \in \mathcal{M}\left(X_{1}, T_{1}\right)\right\} \\
& =P^{\mathbf{a}}\left(T_{1}, \sum_{I \in \mathcal{L}_{n}\left(X_{1}\right)} q(I) \Phi_{I}\right),
\end{aligned}
$$

where $\chi_{[I]}$ denotes the indicator function of $[I]$, and $\Phi_{I}$ denotes the additive potential $\left(\sum_{i=0}^{m-1} \chi_{[I]}\left(T_{1}^{i} x\right)\right)_{m=1}^{\infty}$. By Proposition 4.1, $g^{*}$ is differentiable over $\mathbb{R}^{\mathcal{L}_{n}\left(X_{1}\right)}$ and

$$
\nabla g^{*}(\mathbf{q})=\left(\left(\Phi_{I}\right)_{*}\left(\mu_{\mathbf{q}}\right)\right)_{I \in \mathcal{A}^{n}}=\left(\mu_{\mathbf{q}}([I])\right)_{I \in \mathcal{L}_{n}\left(X_{1}\right)},
$$

where $\mu_{\mathbf{q}}$ denotes the unique a-weighted equilibrium state of $\sum_{I \in \mathcal{L}_{n}\left(X_{1}\right)} q(I) \Phi_{I}$. Applying Proposition 4.3(ii) to $g^{*}$, we have

$$
\left\{\nabla g^{*}(\mathbf{q}): \mathbf{q} \in \mathbb{R}^{\mathcal{L}_{n}\left(X_{1}\right)}\right\} \supseteq \operatorname{ri}\left(\operatorname{dom} g^{* *}\right)=\operatorname{ri}(\operatorname{dom} g)=\operatorname{ri}\left(\Omega_{n}\right) .
$$

Now let $\eta \in \mathcal{M}\left(X_{1}, T_{1}\right)$ so that $(\eta([I]))_{I \in \mathcal{L}_{n}\left(X_{1}\right)} \in \operatorname{ri}\left(\Omega_{n}\right)$. Therefore by (4.2), there exists $\mathbf{q} \in \mathbb{R}^{\mathcal{L}_{n}\left(X_{1}\right)}$ so that $\nabla g^{*}(\mathbf{q})=(\eta([I]))_{I \in \mathcal{L}_{n}\left(X_{1}\right)}$; and thus by (4.1), $\left(\mu_{\mathbf{q}}([I])\right)_{I \in \mathcal{L}_{n}\left(X_{1}\right)}=(\eta([I]))_{I \in \mathcal{L}_{n}\left(X_{1}\right)}$. Now assume that $\widetilde{\mu}$ is a $T_{1}$-invariant measure different from $\mu_{\mathbf{q}}$ such that $(\tilde{\mu}(I))_{I \in \mathcal{L}_{n}\left(X_{1}\right)}=(\eta(I))_{I \in \mathcal{L}_{n}\left(X_{1}\right)}$. Since $\mu_{\mathbf{q}}$ is the unique a-weighted equilibrium state of $\sum_{I \in \mathcal{L}_{n}\left(X_{1}\right)} q(I) \Phi_{I}$, we have

$$
\begin{aligned}
P^{\mathbf{a}}\left(T_{1}, \sum_{I \in \mathcal{L}_{n}\left(X_{1}\right)} q(I) \Phi_{I}\right) & =\sum_{I \in \mathcal{L}_{n}\left(X_{1}\right)} q(I) \int \chi_{[I]} d \mu_{\mathbf{q}}+h_{\mu_{\mathbf{q}}}^{\mathbf{a}}\left(T_{1}\right) \\
& >\sum_{I \in \mathcal{L}_{n}\left(X_{1}\right)} q(I) \int \chi_{[I]} d \widetilde{\mu}+h_{\widetilde{\mu}}^{\mathbf{a}}\left(T_{1}\right) .
\end{aligned}
$$

It follows that $h_{\mu_{\mathbf{q}}}^{\mathbf{a}}\left(T_{1}\right)>h_{\widetilde{\mu}}^{\mathbf{a}}\left(T_{1}\right)$. This proves (i).

To prove (ii), let $\eta_{0} \in \mathcal{M}\left(X_{1}, T_{1}\right)$ be the measure having the maximal a-weighted entropy. Clearly, $\eta_{0}$ is the a-weighted equilibrium state of $f \equiv 0$. If $\eta=\eta_{0}$, then 
we just take $\mu_{n}=\eta_{0}$ for $n \geq 1$ and we are done. In the following we assume that $\eta \neq \eta_{0}$. For each $n \in \mathbb{N}$, we pick $\xi_{n} \in \mathcal{M}\left(X_{1}, T_{1}\right)$ such that $\left(\xi_{n}([I])\right)_{I \in \mathcal{L}_{n}\left(X_{1}\right)} \in \operatorname{ri}\left(\Omega_{n}\right)$. Then choose a small $p_{n}>0$ such that $p_{n} h_{\xi_{n}}^{\mathbf{a}}\left(T_{1}\right)+\left(1-p_{n}\right) h_{\eta_{0}}^{\mathbf{a}}\left(T_{1}\right)>h_{\eta}^{\mathbf{a}}\left(T_{1}\right)$. Define $\widetilde{\xi_{n}}=p_{n} \xi_{n}+\left(1-p_{n}\right) \eta_{0}$. Let $\eta_{n}=(1-1 / n) \eta+(1 / n) \widetilde{\xi}_{n}$. Then it is readily checked that $\eta_{n} \in \operatorname{ri}\left(\Omega_{n}\right)$. Let $\mu_{n}=\mu\left(\mathbf{a}, \eta_{n}, n\right)$. Then the sequence $\left(\mu_{n}\right)$ has the desired properties.

口

REMARK 4.4. Let $X_{1}$ be a full shift and $\mu \in \mathcal{M}\left(X_{1}, T_{1}\right)$. Taking $\mathbf{a}=(1, \ldots, 1)$ in Theorem 1.1 and using the upper semi-continuity of the entropy, we see that there is a sequence of quasi-Bernoulli measures $\left(\mu_{n}\right)_{n=1}^{\infty}$ which converges to $\mu$ in the weak-star topology, such that we have $\lim _{n \rightarrow \infty} h_{\mu_{n} \circ \tau_{i-1}^{-1}}\left(T_{i}\right)=h_{\mu \circ \tau_{i-1}^{-1}}\left(T_{i}\right)$ for all $1 \leq i \leq k$. Moreover, one can deduce from Theorem 3.5 that for any $\mathbf{a}=\left(a_{1}, \ldots, a_{k}\right)$ with $a_{1}>0$ and $a_{i} \geq 0$ for $i \geq 2$, each invariant quasi-Bernoulli measure is the a-weighted equilibrium state of some almost additive potential satisfying the bounded distortion property.

In the remainder of this section, we prove our statement in Example 1.2. Let $X_{1}=\{a, b, c\}^{\mathbb{N}}$ and $X_{2}=\{1,2\}^{\mathbb{N}}$. Let $\pi: X_{1} \rightarrow X_{2}$ be a one-block factor map induced by $a, b \mapsto 1$ and $c \mapsto 2$. We first prove the following lemma.

Lemma 4.5. Let $\eta$ be a fully supported one-step Markov measure on $X_{1}$. If

$$
\frac{\eta([a c])}{\eta([a])}=\frac{\eta([b c])}{\eta([b])} \quad \text { or } \quad \frac{\eta([c a])}{\eta([a])}=\frac{\eta([c b])}{\eta([b])},
$$

then $\eta \circ \pi^{-1}$ is a one-step Markov measure on $X_{2}$; otherwise $\eta \circ \pi^{-1}$ is not a Markov measure of any order.

Proof. Our argument is inspired by the work of Chazottes and Ugalde [13]. Denote $p_{i, j}=\eta([i j]) / \eta([i])$ for $i, j \in\{a, b, c\}$. Clearly

$$
\eta\left(\left[x_{1} x_{2} \ldots x_{n}\right]\right)=\eta\left(\left[x_{1}\right]\right) p_{x_{1}, x_{2}} \ldots p_{x_{n-1}, x_{n}}, \quad \forall x_{1} \ldots x_{n} \in\{a, b, c\}^{n} .
$$

According to (4.4), it is direct to check that

$$
\eta \circ \pi^{-1}\left(\left[y_{1} y_{2} \ldots y_{n}\right]\right)=\mathbf{v}_{y_{1}} M_{y_{1} y_{2}} \ldots M_{y_{n-1} y_{n}} \mathbf{u}_{y_{n}}^{T}, \quad \forall y_{1} \ldots y_{n} \in\{1,2\}^{n},
$$

where

$$
\begin{aligned}
& \mathbf{v}_{1}=(\eta([a]), \eta([b])), \quad \mathbf{v}_{2}=\eta([c]), \quad \mathbf{u}_{1}=(1,1), \quad \mathbf{u}_{2}=1 . \\
& M_{11}=\left(\begin{array}{ll}
p_{a, a} & p_{a, b} \\
p_{b, a} & p_{b, b}
\end{array}\right), \quad M_{12}=\left(\begin{array}{c}
p_{a c} \\
p_{b c}
\end{array}\right), \quad M_{21}=\left(p_{c a}, p_{c b}\right), \quad M_{22}=p_{c c} .
\end{aligned}
$$

Note that $\eta \circ \pi^{-1}$ is a $(k-1)$-step Markov measure if and only if

$$
\frac{\eta \circ \pi^{-1}\left(\left[y_{1} y_{2} \ldots y_{n}\right]\right)}{\eta \circ \pi^{-1}\left(\left[y_{2} \ldots y_{n}\right]\right)} \text { depends only on } y_{1} \ldots y_{k} \text { when } n \geq k \text {. }
$$

However, it is easy to check

$$
\frac{\eta \circ \pi^{-1}\left(\left[y_{1} y_{2} \ldots y_{n}\right]\right)}{\eta \circ \pi^{-1}\left(\left[y_{2} \ldots y_{n}\right]\right)}=\frac{\eta \circ \pi^{-1}\left(\left[y_{1} y_{2} \ldots y_{i}\right]\right)}{\eta \circ \pi^{-1}\left(\left[y_{2} \ldots y_{i}\right]\right)},
$$

provided that $y_{i}=2$ for some $2 \leq i \leq n$. Thus to check whether (4.5) holds for all $y_{1} y_{2} \ldots y_{n}$, it suffices to check the property for the words $1^{n}, 1^{n} 2,21^{n}, 21^{n} 2$. However, 
it is direct to check that (4.5) holds for the words $1^{n}, 1^{n} 2,21^{n}, 21^{n} 2$ for some $k \geq 2$ if and only if $\mathbf{v}_{1}$ and $M_{21}$ are the left eigenvectors of $M_{11}$, or, $M_{21}$ and $\mathbf{u}_{1}$ are the right eigenvectors of $M_{11}$; and this holds if and only if (4.3) holds. In the end, if (4.3) holds then, (4.5) holds for $k=2$. This finishes the proof of the lemma.

Proof of Example 1.2. Let $\eta \in \mathcal{M}\left(X_{1}, T_{1}\right)$ be fully supported. Write $\mu=$ $\mu(\mathbf{a}, \eta, 2)$. By Theorem 1.1, $\mu$ is the a-weighted equilibrium state of some 2-symbol function $f$ on $X_{1}$. Define $\phi^{(0)}:\{a, b, c\}^{*} \rightarrow(0, \infty)$ and $\phi^{(1)}:\{1,2\}^{*} \rightarrow(0, \infty)$ respectively by

$$
\begin{gathered}
\phi^{(0)}(I)=\sup _{x \in[I]} \exp \left(S_{n} f(x)\right) \text { for } I \in\{a, b, c\}^{n} \text { and } \\
\phi^{(1)}(J)=\left(\sum_{I \in \pi^{-1}(J)} \phi^{(0)}(I)^{1 / a_{1}}\right)^{a_{1}} \text { for } J \in\{1,2\}^{n},
\end{gathered}
$$

here and afterwards, $S_{n} f(x)$ denotes the sum $\sum_{i=0}^{n-1} f\left(T_{1}^{i} x\right)$. Then by Theorem 3.5,

$$
\mu([I]) \approx \exp \left(-\frac{n P}{a_{1}+a_{2}}\right) \phi^{(0)}(I)^{\frac{1}{a_{1}}} \phi^{(1)}(\pi I)^{\frac{1}{a_{1}+a_{2}}-\frac{1}{a_{1}}}, \quad I \in\{a, b, c\}^{*},
$$

and

$$
\mu \circ \pi^{-1}([J]) \approx \exp \left(-\frac{n P}{a_{1}+a_{2}}\right) \phi^{(1)}(J)^{\frac{1}{a_{1}+a_{2}}}, \quad J \in\{1,2\}^{*},
$$

where $P=P^{\mathbf{a}}\left(T_{1}, f\right)$. Let $m$ be the equilibrium state of $\frac{1}{a_{1}} f$. Since $\frac{1}{a_{1}} f$ is a 2 -symbol function, $m$ is a one-step Markov measure on $X_{1}$. Clearly

$$
m([I]) \approx \exp (-n Q) \phi^{(0)}(I)^{1 / a_{1}}, \quad m \circ \pi^{-1}([I]) \approx \exp (-n Q) \phi^{(1)}(\pi I)^{1 / a_{1}}
$$

for $I \in\{a, b, c\}^{*}$, where $Q:=P\left(T_{1},\left(1 / a_{1}\right) f\right)$. By Lemma $4.5, m \circ \pi^{-1}$ is either a one-step Markov measure, or not a Markov measure of any order. Hence, either there exists a 2-symbol function $h$ on $X_{2}$ such that

$$
\phi^{(1)}(J) \approx \sup _{y \in[J]} \exp \left(S_{n} h(y)\right), \quad J \in\{1,2\}^{n},
$$

or (4.8) does not hold for any finite-symbol function $h$ on $X_{2}$.

Assume that $\mu$ is a Markov measure of finite order. Then there exists a finitesymbol function $g$ on $X_{1}$ such that $\mu([I]) \approx \sup _{x \in[I]} \exp \left(S_{n} g(x)\right)$ for $I \in\{a, b, c\}^{n}$. Combining this with (4.6) yields that there exists $k \geq 2$ such that (4.8) holds for some $k$-symbol function $h$ on $X_{2}$. Therefore (4.8) holds for some 2-symbol function $\widetilde{h}$ on $X_{2}$. Applying this to (4.6) and (4.7), we see that both $\mu$ and $\mu \circ \pi^{-1}$ are one-step Markov measures. By Lemma 4.5, (1.4) must hold (noting that $\mu([I])$ and $\eta([I])$ coincide for all 2-nd cylinders $[I]$ ).

Conversely, assume that (1.4) holds. Define a measure $\widetilde{\mu}$ on $X_{1}$ by

$$
\widetilde{\mu}\left(\left[x_{1} x_{2} \ldots x_{n}\right]\right)=\eta\left(\left[x_{1}\right]\right) p_{x_{1}, x_{2}} \ldots p_{x_{n-1}, x_{n}}, \quad \forall x_{1} \ldots x_{n} \in\{a, b, c\}^{n},
$$

where $p_{i, j}=\eta([i j]) / \eta([i])$ for $i, j \in\{a, b, c\}$. Then $\widetilde{\mu}$ is a one-step Markov measure so that $\widetilde{\mu}([I])$ and $\eta([I])$ coincide for all 2-nd cylinders $[I]$. By Lemma $4.5, \widetilde{\mu} \circ \pi^{-1}$ is also a one-step Markov measure. Hence $h_{\widetilde{\mu}}\left(T_{1}\right) \geq h_{\widetilde{\eta}}\left(T_{1}\right)$ and $h_{\widetilde{\mu} \circ \pi^{-1}}\left(T_{2}\right) \geq h_{\widetilde{\eta} \circ \pi^{-1}}\left(T_{2}\right)$ for any $\widetilde{\eta} \in \mathcal{M}\left(X_{1}, T_{1}\right)$ so that $\widetilde{\eta}([I])$ and $\eta([I])$ coincide for all 2-nd cylinders $[I]$ (cf. $[19$, Propositions 1-2]). Therefore $\widetilde{\mu}=\mu(\mathbf{a}, \eta, 2)$. 


\section{Proofs of Theorems 1.3 and 1.4 .}

5.1. Preliminary lemmas. Let us introduce some more notation and give useful preliminary facts.

For $1 \leq i \leq k$ and $n \in \mathbb{N}$, let

$$
\ell_{i}(n)=\min \left\{p \in \mathbb{N}: p \geq\left(a_{1}+\cdots+a_{i}\right) n / a_{1}\right\}
$$

and by convention set $\ell_{0}(n)=0$. It is easy to check that

LEMMA 5.1. In $\left(X_{1}, d_{\mathbf{a}}\right)$, the closed ball centered at $x$ of radius $e^{-n / a_{1}}$ is given by

$$
B\left(x, e^{-n / a_{1}}\right)=\left\{y \in X_{1}: \tau_{i-1}\left(y_{\mid \ell_{i}(n)}\right)=\tau_{i-1}\left(x_{\mid \ell_{i}(n)}\right) \text { for all } 1 \leq i \leq k\right\}
$$

For convenience, sometimes for a measure $\mu$ on $X_{i}(i=1, \ldots, k)$, we write $\mu(I)=$ $\mu([I])$ for $I \in \mathcal{L}\left(X_{i}\right)$. The following result estimates the value of an a-weighted Gibbs measure on a ball in $\left(X_{1}, d_{\mathbf{a}}\right)$.

LEMmA 5.2. Suppose that $\left(X_{1}, T_{1}\right)$ is a full shift. Let $\Phi=\left(\log \phi_{n}\right)_{n=1}^{\infty} \in$ $\mathcal{C}_{a a}\left(X_{1}, T_{1}\right)$ satisfy the bounded distortion property. Let $\mu$ denote the a-weighted Gibbs measure of $\Phi$. Then, uniformly in $x=\left(x_{i}\right)_{i=1}^{\infty} \in X_{1}$ and $n \geq 1$, we have

$$
\begin{aligned}
\mu\left(B\left(x, e^{-n / a_{1}}\right)\right) & \approx \prod_{i=1}^{k} \mu_{i}\left(\tau_{i-1}\left(x_{\ell_{i-1}(n)+1} \cdots x_{\ell_{i}(n)}\right)\right) \\
& \approx \exp \left(\frac{-n P^{\mathbf{a}}(T, \Phi)}{a_{1}}\right) \phi_{n}(x)^{1 / a_{1}} \prod_{j=1}^{k-1} \frac{\phi^{(j)}\left(\tau_{j}\left(x_{\mid \ell_{j+1}(n)}\right)\right)^{1 / A_{j+1}}}{\phi^{(j)}\left(\tau_{j}\left(x_{\mid \ell_{j}(n)}\right)\right)^{1 / A_{j}}}
\end{aligned}
$$

where $\mu_{i}=\mu \circ \tau_{i-1}^{-1}$ for $1 \leq i \leq k$, and $\phi^{(j)}, j=0, \ldots, k-1$, are defined as in $(3.3)$, $A_{j}=a_{1}+\cdots+a_{j}$ for $1 \leq j \leq k$.

Proof. We first prove (5.1). Let $x=\left(x_{i}\right)_{i=1}^{\infty} \in X_{1}$ and $n \geq 1$. For $i=1, \ldots, k$, write $U_{i}=x_{\ell_{i-1}(n)+1} \cdots x_{\ell_{k}(n)}$. Let $B$ denote $B\left(x, e^{-n / a_{1}}\right)$. By Lemma $5.1, B=\{y$ : $\left.\forall 1 \leq i \leq k, \tau_{i-1}(y) \in \tau_{i-1}\left(\left[U_{1} \ldots U_{i}\right]\right)\right\}$, so

$$
\mu(B)=\sum_{\left(J_{1}, \ldots, J_{k}\right) \in \Theta} \mu\left(J_{1} \cdots J_{k}\right)
$$

where

$$
\Theta:=\left\{\left(J_{1}, \ldots, J_{k}\right) \in \prod_{i=1}^{k} \mathcal{A}_{1}^{\ell_{i}(n)-\ell_{i-1}(n)}: \forall 1 \leq i \leq k, \tau_{i-1}\left(J_{i}\right)=\tau_{i-1}\left(U_{i}\right)\right\} .
$$

Applying $k-1$ times the quasi Bernoulli property of $\mu$ to each term of the above sum, we get

$$
\mu(B) \approx \sum_{\left(J_{1}, \ldots, J_{k}\right) \in \Theta} \mu\left(J_{1}\right) \cdots \mu\left(J_{k}\right)
$$

and (5.1) follows by summing over $J_{k}, \ldots, J_{2}$ successively. 
Now we prove (5.2). Let us transform (5.1) by using (3.6). Since each word $U_{i}$ is of length $\ell_{i}(n)-\ell_{i-1}(n)$ and by construction $\ell_{k}(n) / A_{k}-n / a_{1}=O(1 / n)$, (3.6) yields

$$
\begin{aligned}
\mu(B) & \approx \exp \left(\frac{-\ell_{k}(n) P^{\mathbf{a}}\left(T_{1}, \Phi\right)}{A_{k}}\right) \prod_{i=1}^{k} \phi^{(i-1)}\left(\tau_{i-1} U_{i}\right)^{1 / A_{i}} \prod_{j=i}^{k-1} \phi^{(j)}\left(\tau_{j} U_{i}\right)^{1 / A_{j+1}-1 / A_{j}} \\
& \approx \exp \left(\frac{-n P^{\mathbf{a}}\left(T_{1}, \Phi\right)}{a_{1}}\right)\left(\prod_{i=0}^{k-1} \phi^{(i)}\left(\tau_{i} U_{i+1}\right)^{1 / A_{i}}\right) \prod_{j=1}^{k-1} \prod_{i=1}^{j} \phi^{(j)}\left(\tau_{j} U_{i}\right)^{1 / A_{j+1}-1 / A_{j}} \\
& \approx \exp \left(\frac{-n P^{\mathbf{a}}\left(T_{1}, \Phi\right)}{a_{1}}\right) \phi^{(0)}\left(U_{1}\right)^{1 / a_{1}} \prod_{j=1}^{k-1} \frac{\phi^{(j)}\left(\tau_{j}\left(U_{1} \cdots U_{j+1}\right)\right)^{1 / A_{j+1}}}{\phi^{(j)}\left(\tau_{j}\left(U_{1} \cdots U_{j}\right)\right)^{1 / A_{j}}} \\
& \approx \exp \left(\frac{-n P^{\mathbf{a}}\left(T_{1}, \Phi\right)}{a_{1}}\right) \phi_{n}(x)^{1 / a_{1}} \prod_{j=1}^{k-1} \frac{\phi^{(j)}\left(\tau_{j} x_{\mid \ell_{j+1}(n)}\right)^{1 / A_{j+1}}}{\phi^{(j)}\left(\tau_{j} x_{\mid \ell_{j}(n)}\right)^{1 / A_{j}}} .
\end{aligned}
$$

This finishes the proof of the lemma.

5.2. Proof of Theorem 1.3. The main result of this section is the following.

THEOREM 5.3. Suppose that $X_{1}$ satisfies the specification property. Let $\mu \in$ $\mathcal{M}\left(X_{1}, T_{1}\right)$. Then $\operatorname{dim}_{H} G_{\mu}\left(X_{1}, T_{1}\right) \geq h_{\mu}^{\mathbf{a}}\left(T_{1}\right)$.

Proof of Theorem 1.3. By Theorem 5.3, to complete the proof of Theorem 1.3 it remains to control $\operatorname{dim}_{H} G_{\mu}\left(X_{1}, T_{1}\right)$ from above.

For any $\varphi \in C\left(X_{1}\right)$, denote $\mu(\varphi)=\int \varphi d \mu$. Furthermore for $\alpha \in \mathbb{R}$, denote

$$
E_{\varphi}(\alpha)=\left\{x \in X_{1}: \lim _{n \rightarrow \infty} \frac{1}{n} S_{n} \varphi(x)=\alpha\right\} .
$$

Then by (1.3), $G_{\mu}\left(X_{1}, T_{1}\right)=\bigcap_{\varphi \in C\left(X_{1}\right)} E_{\varphi}(\mu(\varphi))$. Thus, by using Lemma 5.6 whose proof is independent of the present one, we obtain

$$
\begin{aligned}
\operatorname{dim}_{H} G_{\mu}\left(X_{1}, T_{1}\right) & \leq \inf _{\varphi \in C\left(X_{1}\right)} \operatorname{dim}_{H} E_{\varphi}(\mu(\varphi)) \\
& \leq \inf _{\varphi \in C\left(X_{1}\right)} \inf _{q \in \mathbb{R}}\left(P^{\mathbf{a}}\left(T_{1}, q \varphi\right)-q \mu(\varphi)\right) \\
& =\inf _{q \in \mathbb{R}} \inf _{\varphi \in C\left(X_{1}\right)}\left(P^{\mathbf{a}}\left(T_{1}, q \varphi\right)-q \mu(\varphi)\right) \\
& =\inf _{\varphi \in C\left(X_{1}\right)}\left(P^{\mathbf{a}}\left(T_{1}, \varphi\right)-\mu(\varphi)\right) .
\end{aligned}
$$

Now we note that, on the one hand, the a-weighted topological pressure is the Legendre-Fenchel transform of the a-weighted entropy defined on the compact convex set $\mathcal{M}\left(X_{1}, T_{1}\right)$ of $C(X)^{*}$ endowed with the weak-star topology, and on the other hand, the a-weighted entropy is upper semi-continuous. Hence we have $\inf _{\varphi \in C\left(X_{1}\right)}\left(P^{\mathbf{a}}\left(T_{1}, \varphi\right)-\mu(\varphi)\right)=h_{\mu}^{\mathbf{a}}\left(T_{1}\right)$ by mimicking the proof of Theorem 3.12 in [43]. This yields the conclusion.

Let $\left(\mathcal{A}_{1}^{\mathbb{N}}, T_{1}\right)$ denote the full shift over the alphabet $\mathcal{A}_{1}$. Then $X_{1}$ can be viewed as an $T_{1}$-invariant subset of $\mathcal{A}_{1}^{\mathbb{N}}$. For $p \in \mathbb{N}$, write $\mathcal{L}_{p}:=\mathcal{L}_{p}\left(X_{1}\right)$ for short, where $\mathcal{L}_{p}\left(X_{1}\right)$ denotes the $p$-th language of $X_{1}$ (cf. Section 2.1). We use $\left(\mathcal{L}_{p}^{\mathbb{N}}, T_{1}^{p}\right)$ to denote the full shift over the alphabet $\mathcal{L}_{p}$. Again $\mathcal{L}_{p}^{\mathbb{N}}$ can be viewed as a subset $\mathcal{A}_{1}^{\mathbb{N}}$, and $X_{1} \subset \mathcal{L}_{p}^{\mathbb{N}}$. 
Proof of Theorem 5.3. Let us outline the main steps in our approach. Fix $\mu \in$ $\mathcal{M}\left(X_{1}, T_{1}\right)$. We first construct a set $G_{\mu} \subset \mathcal{A}_{1}^{\mathbb{N}}$ such that

$$
G_{\mu} \subset G_{\mu}\left(\mathcal{A}_{1}^{\mathbb{N}}, T_{1}\right) \text { and } \operatorname{dim}_{H} G_{\mu} \geq h_{\mu}^{\mathbf{a}}\left(T_{1}\right),
$$

where $\mu$ is considered as an element of $\mathcal{M}\left(\mathcal{A}_{1}^{\mathbb{N}}, T_{1}\right)$. Once this is done, we will define an injective map conc : $G \rightarrow X_{1}$ such that $\operatorname{conc}\left(G_{\mu}\right) \subset G_{\mu}\left(X_{1}, T_{1}\right)$ and $\operatorname{dim}_{H} \operatorname{conc}\left(G_{\mu}\right)=\operatorname{dim}_{H} G_{\mu}$. Hence $G_{\mu}\left(X_{1}, T_{1}\right) \geq \operatorname{dim}_{H} G_{\mu} \geq h_{\mu}^{\mathbf{a}}\left(T_{1}\right)$.

Now we start the rigorous construction of $G_{\mu}$. Pick a countable family of Hölder continuous functions

$$
\widetilde{\mathcal{C}}=\left\{\varphi_{m}: m=1,2, \ldots\right\} \subset C\left(\mathcal{A}_{1}^{\mathbb{N}}\right)
$$

so that it is dense in $C\left(\mathcal{A}_{1}^{\mathbb{N}}\right)$. Next we construct a sequence $\left(\mu_{p}\right)$ of measures on $\mathcal{A}_{1}^{\mathbb{N}}$ as below.

Let $p \in \mathbb{N}$. Since $X_{1} \subset \mathcal{L}_{p}^{\mathbb{N}}$, the measure $\mu$ has a trivial extension to the Borel subsets of $\mathcal{L}_{p}^{\mathbb{N}}$ defined by $A \mapsto \mu\left(A \cap X_{1}\right)$. We still denote this extension by $\mu$; it is $T_{1}^{p}$ invariant. As $\mathcal{L}_{p}^{\mathbb{N}}$ is a full shift, by Remark $4.4, \mu$ is the limit in the weak-star topology of a sequence of quasi-Bernoulli measures $\left(\eta_{j}\right)_{j \geq 1}$ on $\left(\mathcal{L}_{p}^{\mathbb{N}}, T_{1}^{p}\right)$ such that $h_{\eta_{j} \circ \tau_{i-1}^{-1}}\left(T_{i}^{p}\right)$ converges to $h_{\mu \circ \tau_{i-1}^{-1}}\left(T_{i}^{p}\right)=p h_{\mu \circ \tau_{i-1}^{-1}}\left(T_{i}\right)$ for all $1 \leq i \leq k$, and $\eta_{j}\left(S_{p} \varphi_{m}\right)$ converges to $\mu\left(S_{p} \varphi_{m}\right)=p \mu\left(\varphi_{m}\right)$ for all $1 \leq m \leq p$, as $j \rightarrow \infty$. Hence we may pick a large enough $j$, by setting $\mu_{p}=\eta_{j}$, we have

$$
\begin{aligned}
& \left|h_{\mu_{p} \circ \tau_{i-1}^{-1}}\left(T_{i}^{p}\right)-p h_{\mu \circ \tau_{i-1}^{-1}}\left(T_{i}\right)\right| \leq \frac{1}{2 p}, \quad \forall 1 \leq i \leq k, \\
& \left|\mu_{p}\left(S_{p} \varphi_{m}\right)-p \mu\left(\varphi_{m}\right)\right| \leq \frac{1}{2 p}, \quad 1 \leq m \leq p .
\end{aligned}
$$

As above, we can obtain a sequence $\left(\mu_{p}\right)_{p=1}^{\infty}$ so that (5.4) holds for each $p \in \mathbb{N}$; moreover $\mu_{p}$ is quasi-Bernoulli and ergodic on $\left(\mathcal{L}_{p}^{\mathbb{N}}, T_{1}^{p}\right)$, and it can be viewed as a $T_{1}^{p}$ invariant and ergodic measure on $\mathcal{A}_{1}^{\mathbb{N}}$. Let $\left(\kappa_{p}\right)$ be an increasing sequence of positive numbers such that

$$
\kappa_{p}^{-1} \mu_{p}([I]) \mu_{p}([J]) \leq \mu_{p}([I J]) \leq \kappa_{p} \mu_{p}([I]) \mu_{p}([J]), \quad \forall I, J \in \bigcup_{i=0}^{\infty} \mathcal{L}_{p}^{i} .
$$

For $p, N \in \mathbb{N}$, let $G(p, N)$ denote the set of points $x \in \mathcal{L}_{p}^{\mathbb{N}} \subset \mathcal{A}_{1}^{\mathbb{N}}$ such that

$$
\begin{aligned}
& \left|\frac{\log \mu_{p} \circ \tau_{i-1}^{-1}\left(\tau_{i-1} x_{\mid p n}\right)}{-n}-p h_{\mu \circ \tau_{i-1}^{-1}}\left(T_{i}\right)\right| \leq \frac{1}{p} \quad \text { for } n \geq N \text { and } 1 \leq i \leq k, \text { and } \\
& \left|\frac{S_{n p} \varphi_{m}(x)}{n}-p \mu\left(\varphi_{m}\right)\right| \leq \frac{1}{p} \quad \text { for } n \geq N \text { and } 1 \leq m \leq p .
\end{aligned}
$$

By using the Shannon-McMillian-Brieman theorem (cf. [44, p. 93]) and the Birkhoff ergodic theorem to the ergodic measure dynamic systems $\left(\mathcal{A}_{i}^{\mathbb{N}}, T_{i}^{p}, \mu_{p} \circ \tau_{i-1}\right)(1 \leq i \leq$ $k)$, we have for $\mu_{p}$-a.e. $x$,

$$
\begin{gathered}
\lim _{n \rightarrow \infty} \frac{1}{n} \log \mu_{p} \circ \tau_{i-1}^{-1}\left(\tau_{i-1} x_{\mid p n}\right)=-h_{\mu_{p}}\left(T_{i}^{p}\right), \quad i=1, \ldots, k, \text { and } \\
\lim _{n \rightarrow \infty} \frac{1}{n} S_{n p} \varphi_{m}(x)=\lim _{n \rightarrow \infty} \frac{1}{n} \sum_{i=0}^{n-1}\left(S_{p} \varphi_{m}\right)\left(T_{1}^{p i} x\right)=\mu_{p}\left(S_{p} \varphi_{m}\right), \quad 1 \leq m \leq p .
\end{gathered}
$$


Combining them with (5.4) yields

$$
\lim _{N \rightarrow \infty} \mu_{p}(G(p, N))=1 .
$$

Hence we can choose a sequence $\left(N_{p}\right)$ of increasing positive integers such that

$$
\mu_{p}\left(G\left(p, N_{p}\right)\right) \geq 1-2^{-p}, \quad p=1,2, \ldots .
$$

Next construct a sequence $\left(M_{p}\right)$ of positive integers such that

$$
M_{p} \geq \max \left\{2^{M_{p-1}}, \kappa_{p}, 2^{N_{p+1}}\right\}, \quad p=2,3, \ldots
$$

and set

$$
N_{p}^{\prime}=N_{p} \times M_{p}, \quad p=1,2, \ldots
$$

Then, for $p \geq 1$ let $\widetilde{\mu}_{p}$ be the discrete measure on $\mathcal{A}_{1}^{p N_{p}^{\prime}}$ defined by

$$
\widetilde{\mu}_{p}(I)=\mu_{p}([I]) .
$$

Clearly, $\widetilde{\mu}_{p}$ is supported on $\mathcal{L}_{p}^{N_{p}^{\prime}}$. Now we define

$$
\begin{aligned}
G & =\prod_{p=1}^{\infty} \mathcal{L}_{p}^{N_{p}^{\prime}}, \quad \nu=\otimes_{p=1}^{\infty} \widetilde{\mu}_{p}, \\
G_{p} & =\left\{I \in \mathcal{L}_{p}^{N_{p}^{\prime}}:[I] \cap G\left(p, N_{p}\right) \neq \emptyset\right\}, \quad p=1,2, \ldots
\end{aligned}
$$

Finally, we define

$$
G_{\mu}=\otimes_{p \geq 1} G_{p}=\left\{I_{1} I_{2} \cdots I_{p} \cdots \in \mathcal{A}_{1}^{\mathbb{N}}: \forall p \geq 1, I_{p} \in G_{p}\right\} .
$$

By construction, the measure $\nu$ is supported on $G$ and we have

$$
\nu\left(G_{\mu}\right)=\prod_{p \geq 1} \widetilde{\mu}_{p}\left(G_{p}\right) \geq \prod_{p \geq 1} \mu_{p}\left(G\left(p, N_{p}\right)\right) \geq \prod_{p \geq 1}\left(1-2^{-p}\right)>0 .
$$

Now we prove that (5.3) holds for $G_{\mu}$. It is enough to show that

$$
\begin{gathered}
G_{\mu} \subset G_{\mu}\left(\mathcal{A}_{1}^{\mathbb{N}}, T_{1}\right) \text { and } \\
\liminf _{n \rightarrow \infty} \frac{\log \nu\left(B\left(x, e^{-n / a_{1}}\right)\right)}{-n / a_{1}} \geq h_{\mu}^{\mathbf{a}}\left(T_{1}\right) \quad \text { for all } x \in G_{\mu}
\end{gathered}
$$

since (5.10) implies $\operatorname{dim}_{H} G_{\mu} \geq h_{\mu}^{\mathbf{a}}\left(T_{1}\right)$ (see [18] for instance).

Proof of (5.9). Note that the sequence $\left(\varphi_{m}\right)$ is dense in $C\left(\mathcal{A}_{1}^{\mathbb{N}}\right)$, it suffices to show that for each $x \in G_{\mu}$ and $m \in \mathbb{N}$,

$$
\lim _{n \rightarrow \infty} \frac{1}{n} S_{n} \varphi_{m}(x)=\mu\left(\varphi_{m}\right) .
$$

Now we fix such a pair $x, m$, and write $\varphi=\varphi_{m}$. Write $x$ in the concatenated form:

$$
x=I_{1} I_{2} \ldots I_{p} \ldots, \quad I_{j} \in G_{j} \subset \mathcal{L}_{j}^{N_{j}^{\prime}} \text { for } j \geq 1 .
$$


For a large number $n$, let $p$ be the unique integer so that $\sum_{j=1}^{p-1} j N_{j}^{\prime}<n \leq$ $\sum_{j=1}^{p} j N_{j}^{\prime}$. That is, $p$ is the integer such that the $n$-th digit of $x$ is located in the word $I_{p}$. Furthermore let $q$ be the unique integer $q \in\left[1, M_{p}\right]$ such that

$$
\sum_{j=1}^{p-1} j N_{j}^{\prime}+(q-1) p N_{p}<n \leq \sum_{j=1}^{p-1} j N_{j}^{\prime}+q p N_{p} .
$$

That is, if we write $I_{p}=W_{1} W_{2} \ldots W_{M_{p}}$ with $W_{i} \in \mathcal{L}_{p}^{N_{p}}$, then the $n$-th digit of $x$ is located in the word $W_{q}$. The condition (5.7) guarantees that

$$
\sum_{j=1}^{p-2} j N_{j}^{\prime}=o\left((p-1) N_{p-1}^{\prime}\right)=o(n), \quad p N_{p}=o(n) .
$$

Hence

$$
S_{n} \varphi(x)=S_{(p-1) N_{p-1}^{\prime}} \varphi\left(T_{1}^{u} x\right)+S_{q p N_{p}} \varphi\left(T_{1}^{u+(p-1) N_{p-1}^{\prime}} x\right)+o(n),
$$

where $u:=\sum_{j=1}^{p-2} j N_{j}^{\prime}$. Keep in mind that $T_{1}^{u} x \in\left[I_{p-1}\right]$ and $T_{1}^{u+(p-1) N_{p-1}^{\prime}} x \in\left[I_{p}\right]$. Since $I_{j} \in G_{j}$, by the construction (5.8), $\left[I_{j}\right] \cap G\left(j, N_{j}\right) \neq \emptyset$ for $j=p-1, p$. Hence, by (5.6), there exist $y \in\left[I_{p-1}\right]$ and $z \in\left[I_{p}\right]$ such that

$$
S_{(p-1) N_{p-1}^{\prime}} \varphi(y)=(p-1) N_{p-1}^{\prime} \mu(\varphi)+o(n), \quad S_{q p N_{p}} \varphi(z)=q p N_{p} \mu(\varphi)+o(n) .
$$

Since $\varphi$ is Hölder continuous, it has the following bounded distortion property

$$
\begin{aligned}
& S_{(p-1) N_{p-1}^{\prime}} \varphi\left(T_{1}^{u} x\right)=S_{(p-1) N_{p-1}^{\prime}} \varphi(y)+O(1), \\
& S_{q p N_{p}} \varphi\left(T_{1}^{u+(p-1) N_{p-1}^{\prime}} x\right)=S_{q p N_{p}} \varphi(z)+O(1) .
\end{aligned}
$$

Combining (5.11)-(5.13) yields

$$
S_{n} \varphi(x)=\left((p-1) N_{p-1}^{\prime}+q p N_{p}\right) \mu(\varphi)+o(n)=n \mu(\varphi)+o(n) .
$$

That is, $\lim _{n \rightarrow \infty} \frac{1}{n} S_{n} \varphi(x)=\mu(\varphi)$. This finishes the proof of (5.9).

Proof of (5.10). Fix $x \in G_{\mu}$. Write $x$ in the concatenated form:

$$
x=I_{1} I_{2} \ldots I_{p} \ldots, \quad I_{j} \in G_{j} \subset \mathcal{L}_{j}^{N_{j}^{\prime}} \text { for } j \geq 1 .
$$

Let $n$ be a large integer. To estimate $\nu\left(B\left(x, e^{-n / a_{1}}\right)\right)$, recall that (cf. Lemma 5.1)

$$
B\left(x, e^{-n / a_{1}}\right)=\left\{y \in X_{1}: \tau_{i-1}\left(y_{\mid \ell_{i}(n)}\right)=\tau_{i-1}\left(x_{\mid \ell_{i}(n)}\right) \text { for all } 1 \leq i \leq k\right\} .
$$

where

$$
\ell_{i}(n)=\min \left\{p \in \mathbb{N}: p \geq\left(a_{1}+\cdots+a_{i}\right) n / a_{1}\right\},
$$

in particular, $\ell_{1}(n)=n$. For convention write $\ell_{0}(n)=0$.

For $i=1, \ldots, k$, let $p_{i}$ denote the unique integer such that the $\ell_{i}(n)$-th digit of $x$ is located in the word $I_{p_{i}}$. Note that $p_{i}$ depends only on $n$ and $i$, and is independent of $x$. Due to (5.7), $\left|I_{1} I_{2} \ldots I_{p-1}\right|=o\left(\left|I_{p}\right|\right)$, where $|I|$ denotes the length of the word $I$. Hence there are only two possibilities when $n$ is large enough: 
(C1) $p_{1}=p_{2}=\ldots=p_{k}(=: p)$;

(C2) there exists $2 \leq \iota \leq k$ so that $p_{1}=\ldots=p_{\iota-1}(=: p), p_{\iota}=\ldots=p_{k}=p+1$;

Without loss of generality we assume that $a_{i}>0$ for all $1 \leq i \leq k$.

First we consider the case $(\mathrm{C} 1)$. Write the word $I_{p}$ in the following form

$$
I_{p}=U_{1} W_{1} U_{2} W_{2} \ldots U_{k} W_{k} U_{k+1}
$$

in a way such that $\left|W_{i}\right|=p N_{p}$ for $i=1, \ldots, k$ and $\left|U_{j}\right|$ are multiples of $p N_{p}$ (maybe 0 ) for $j=1, \ldots k+1$, and in particular, the $\ell_{i}(n)$-th digit of $x$ is located in the word $W_{i}$ for $i=1, \ldots, k$. Clearly, the above decomposition of $I_{p}$ is unique (i.e., $\left|U_{j}\right|$ 's are uniquely determined). Now we consider the following concatenation

$$
x=I_{1} I_{2} \ldots I_{p-1} U_{1} W_{1} U_{2} W_{2} \ldots U_{k} W_{k} U_{k+1} I_{p+1} \ldots
$$

Due to (5.7), we have

$$
\begin{aligned}
& \left|I_{1} I_{2} \ldots I_{p-2}\right|=o\left(\left|I_{p-1}\right|\right)=o(n), \quad\left|W_{i}\right|=o(n), \\
& \left|I_{p-1}\right|+\left|U_{1}\right|=\ell_{1}(n)+o(n), \quad\left|U_{j}\right|=\ell_{j}(n)-\ell_{j-1}(n)+o(n)
\end{aligned}
$$

for $i=1, \ldots, k$ and $2 \leq j \leq k$.

By the construction of $\nu$ (cf. (5.8)), and the quasi-Bernoulli property of $\mu_{p}$ (cf. (5.5)), we have the following estimation

$$
\nu\left(\left[x_{\mid \ell_{k}(n)}\right]\right) \leq \kappa_{p}^{k} \nu\left(\left[I_{1} \ldots I_{p-2}\right]\right) \mu_{p-1}\left(\left[I_{p-1}\right]\right) \prod_{i=1}^{k} \mu_{p}\left(\left[U_{i}\right]\right) .
$$

Similarly for any $y \in G=\prod_{j=1}^{\infty} \mathcal{L}_{j}^{N_{j}^{\prime}}$, if we write $y$ in the same form

$$
y=\widetilde{I}_{1} \widetilde{I}_{2} \ldots \widetilde{I}_{p-1} \widetilde{U}_{1} \widetilde{W}_{1} \widetilde{U}_{2} \widetilde{W}_{2} \ldots \widetilde{U}_{k} \widetilde{W}_{k} \widetilde{U}_{k+1} \widetilde{I}_{p+1} \ldots,
$$

we have

$$
\nu\left(\left[y_{\mid \ell_{k}(n)}\right]\right) \leq \kappa_{p}^{k} \nu\left(\left[\widetilde{I}_{1} \ldots \widetilde{I}_{p-2}\right]\right) \mu_{p-1}\left(\left[\widetilde{I}_{p-1}\right]\right) \prod_{i=1}^{k} \mu_{p}\left(\left[\widetilde{U}_{i}\right]\right) .
$$

Then by (5.14), we obtain

$$
\begin{aligned}
\nu\left(B\left(x, e^{-n / a_{1}}\right)\right) & \leq \kappa_{p}^{k}\left(\# \mathcal{L}_{p}^{N_{p}}\right)^{k} \nu\left(\left[I_{1} \ldots I_{p-2}\right]\right) \mu_{p-1}\left(\left[I_{p-1}\right]\right) \prod_{i=1}^{k} \mu_{p} \circ \tau_{i-1}^{-1}\left(\left[\tau_{i-1} U_{i}\right]\right) \\
& \leq \kappa_{p}^{k}\left(\# \mathcal{A}_{1}\right)^{p N_{p} k} \mu_{p-1}\left(\left[I_{p-1}\right]\right) \prod_{i=1}^{k} \mu_{p} \circ \tau_{i-1}^{-1}\left(\left[\tau_{i-1} U_{i}\right]\right) .
\end{aligned}
$$

Since $\left[I_{j}\right] \cap G\left(j, N_{j}\right) \neq \emptyset$ for $j=p-1, p$, and $\left|W_{i}\right|=o(n)$, by (5.6), we have

$$
\log \mu_{p-1}\left(\left[I_{p-1}\right]\right)=-(p-1) N_{p-1}^{\prime} h_{\mu}\left(T_{1}\right)+o(n),
$$

and for $i=1, \ldots, k$,

$$
\begin{aligned}
& \log \mu_{p} \circ \tau_{i-1}^{-1}\left(\left[\tau_{i-1}\left(U_{1} W_{1} \ldots U_{i} W_{i}\right)\right]\right)=-\left(\left|U_{1}\right|+\ldots+\left|U_{i}\right|\right) h_{\mu \circ \tau_{i-1}^{-1}}\left(T_{i}\right)+o(n) . \\
& \log \mu_{p} \circ \tau_{i-1}^{-1}\left(\left[\tau_{i-1}\left(U_{1} W_{1} \ldots U_{i-1} W_{i-1}\right)\right]\right)=-\left(\left|U_{1}\right|+\ldots+\left|U_{i-1}\right|\right) h_{\mu \circ \tau_{i-1}^{-1}}\left(T_{i}\right)+o(n) .
\end{aligned}
$$


which implies that

$$
\begin{aligned}
\log \mu_{p} \circ \tau_{i-1}^{-1}\left(\left[\tau_{i-1} U_{i}\right]\right) & =\log \mu_{p} \circ \tau_{i-1}^{-1}\left(\left[\tau_{i-1}\left(U_{i} W_{i}\right)\right]\right)+o(n) \\
& =-\left|U_{i}\right| h_{\mu \circ \tau_{i-1}^{-1}}\left(T_{i}\right)+o(n),
\end{aligned}
$$

by using the quasi-Bernoulli property of $\mu_{p}$ and $\log \kappa_{p}=o(n)$. Combining (5.15)(5.17) yields

$$
\log \nu\left(B\left(x, e^{-n / a_{1}}\right)\right) \leq-\left(\left|I_{p-1}\right|+\left|U_{1}\right|\right) h_{\mu}\left(T_{1}\right)+\sum_{i=2}^{k}\left|U_{i}\right| h_{\mu \circ \tau_{i-1}^{-1}}\left(T_{i}\right)+o(n) .
$$

Hence

$$
\log \nu\left(B\left(x, e^{-n / a_{1}}\right)\right) \leq\left(-n / a_{1}\right) h_{\mu}^{\mathbf{a}}\left(T_{1}\right)+o(n),
$$

because $\left|I_{p-1}\right|+\left|U_{1}\right|=\ell_{1}(n)+o(n)=n+o(n)$ and $\left|U_{i}\right|=\ell_{i}(n)-\ell_{i-1}(n)+o(n)=$ $a_{i} n / a_{1}+o(n)$ for $2 \leq i \leq k$.

Next we consider the case $(\mathrm{C} 2)$. Write the words $I_{p}, I_{p+1}$ as

$$
I_{p}=U_{1} W_{1} U_{2} W_{2} \ldots U_{\iota-1} W_{\iota-1} V_{\iota}, \quad I_{p+1}=U_{\iota} W_{\iota} \ldots U_{k} W_{k} U_{k+1},
$$

so that $\left|W_{i}\right|=p N_{p}$ for $i=1, \ldots, \iota-1$, and $(p+1) N_{p+1}$ for $i=\iota, \ldots, k$; furthermore, $\left|U_{1}\right|, \ldots,\left|U_{\iota-1}\right|,\left|V_{\iota-1}\right|$ are multiples of $p N_{p}$, whilst $\left|U_{j}\right|$ 's are multiples of $(p+1) N_{p+1}$ for $j=\iota, \ldots, k+1$; in particular, the $\ell_{i}(n)$-th digit of $x$ is located in $W_{i}$ for $i=1, \ldots, k$. In this way, we have the following concatenation

$$
x=I_{1} I_{2} \ldots I_{p-1} U_{1} W_{1} U_{2} W_{2} \ldots U_{\iota-1} W_{\iota-1} V_{\iota} \quad U_{\iota} W_{\iota} \ldots U_{k} W_{k} U_{k+1} I_{p+2} \ldots,
$$

for which we have

$\left|I_{1} I_{2} \ldots I_{p-1}\right|=o\left(\left|I_{p}\right|\right)=o(n), \quad\left|U_{i}\right|=\ell_{i}(n)-\ell_{i-1}(n)+o(n)$ for $1 \leq i \leq \iota-1$ and

$\left|V_{\iota}\right|+\left|U_{\iota}\right|=\ell_{\iota}(n)-\ell_{\iota-1}(n), \quad\left|U_{i}\right|=\ell_{i}(n)-\ell_{i-1}(n)+o(n)$ for $\iota+1 \leq i \leq k$,

$\left|W_{i}\right|=o(n)$ for $1 \leq i \leq k$.

Similar to the case $(\mathrm{C} 1)$, we can show that

$$
\begin{aligned}
\nu\left(B\left(x, e^{-n / a_{1}}\right)\right) \leq \kappa_{p}^{k}\left(\# \mathcal{A}_{1}\right)^{p N_{p} k} & \left(\prod_{i=1}^{\iota-1} \mu_{p} \circ \tau_{i-1}^{-1}\left(\left[\tau_{i-1} U_{i}\right]\right)\right) \mu_{p} \circ \tau_{\iota-1}^{-1}\left(\left[\tau_{\iota-1} V_{\iota}\right]\right) \\
& \cdot \prod_{j=\iota}^{k} \mu_{p+1} \circ \tau_{j-1}^{-1}\left(\left[\tau_{j-1} U_{j}\right]\right) .
\end{aligned}
$$

Then by a similar discussion as in case (C1), we obtain the estimation (5.18). This finishes the proof of $(5.10)$.

To end the proof of Theorem 5.3, we need to define an injective map conc : $G \rightarrow X_{1}$ such that $\operatorname{conc}\left(G_{\mu}\right) \subset G_{\mu}\left(X_{1}, T_{1}\right)$ and $\operatorname{dim}_{H} \operatorname{conc}\left(G_{\mu}\right)=\operatorname{dim}_{H} G_{\mu}$. For this purpose, assume that $X_{1}$ satisfies $s$-specification for some integer $s \geq 1$. Then there exists a map $\theta: \mathcal{L}\left(X_{1}\right) \times \mathcal{L}\left(X_{1}\right) \rightarrow \mathcal{L}_{s}$ such that

$$
I \theta(I, J) J \in \mathcal{L}_{|I|+|J|+s}, \quad \forall I, J \in \mathcal{L}\left(X_{1}\right) .
$$


For $x \in G=\prod_{j=1}^{\infty} \mathcal{L}_{j}^{N_{j}^{\prime}}$, write $x$ in the following concatenated form

$$
x=W_{1,1} W_{1,2} \cdots W_{1, N_{1}^{\prime}} W_{2,1} W_{2,2} \cdots W_{2, N_{2}^{\prime}} \cdots W_{p, 1} W_{p, 2} \cdots W_{p, N_{p}^{\prime}} \cdots,
$$

where $W_{i, j} \in \mathcal{L}_{i}$ for $i \geq 1$ and $1 \leq j \leq N_{i}^{\prime}$. Relabel the words $W_{i, j}$ to get

$$
x=W_{1} W_{2} W_{3} \cdots
$$

Then we define

$$
\operatorname{conc}(x)=W_{1} \theta_{1} W_{2} \theta_{2} W_{3} \theta_{3} \cdots,
$$

where the sequence $\left(\theta_{n}\right)$ is defined inductively by $\theta_{1}=\theta\left(W_{1}, W_{2}\right), \theta_{2}=$ $\theta\left(W_{1} \theta_{1} W_{2}, W_{3}\right)$, and once $\theta_{1}, \ldots, \theta_{p-1}$ have been defined, then define $\theta_{p}=$ $\theta\left(W_{1} \theta_{1} W_{2} \ldots \theta_{p-1} W_{p}, W_{p+1}\right)$.

Clearly, $\operatorname{conc}(x) \in X_{1}$, and the map conc $: G \rightarrow X_{1}$ is injective. Since $\left|W_{j}\right| \rightarrow \infty$ as $j \rightarrow \infty$, conc is almost Lipschitz, thus it preserves the Hausdorff dimension (i.e., $\operatorname{dim}_{H} \operatorname{conc}(E)=\operatorname{dim}_{H} E$ for any $\left.E \subseteq G\right)$. Furthermore, $\operatorname{conc}\left(G_{\mu}\right) \subset G_{\mu}\left(X_{1}, T_{1}\right)$. To see this, it is enough to show that if $\lim \frac{1}{n} S_{n} \varphi(x)=\alpha$, then $\lim \frac{1}{n} S_{n} \varphi(\operatorname{conc}(x))=\alpha$. This can be done in a way similar to the proof of Proposition 6 in [19]. Thus we finish the proof of Theorem 5.3.

REMARK 5.4. The specification property used in this paper can be weakened to cover a wider class of systems. For instance, one can ask that there exists $s \in \mathbb{N}$ such that for all $I, J \in \mathcal{L}:=\mathcal{L}\left(X_{1}\right)$, one can find a word $K$ in $\bigcup_{p=0}^{s} \mathcal{L}_{p}$ such that $I K J \in \mathcal{L}$ (this covers transitive subshifts of finite type). A more general situation is that for all $I, J \in \mathcal{L}$, one can find a word $K \in \mathcal{L}$ such that $I K J \in \mathcal{L}$ and $|K|=o(\min (|I|,|J|)$ as $\min (|I|,|J|)$ tends to $\infty[39]$. Alternatively, one can ask that for all $I, J \in \mathcal{L}$, one can write $I=\widetilde{I} \widehat{I}$ and $J=\widehat{J} \widetilde{J}$ so that $\widetilde{I} \widetilde{J} \in \mathcal{L}$ and $|\widehat{I}|+|\widehat{J}|=o(\min (|I|,|J|)$ as $\min (|I|,|J|)$ tends to $\infty[39]$. In all these cases our approach can be used to obtain the same conclusion as in Theorem 5.3. The only difference is that in these cases the mapping conc introduced in the end of the proof of Theorem 5.3 may take a slightly different form, and it has no reason to be one to one. However, this mapping naturally satisfies the property $\operatorname{conc}\left(G_{\mu}\right) \subset G_{\mu}\left(X_{1}, T_{1}\right)$, and a (tedious, that we omit here) combinatoric argument shows that on the one hand, conc is $\alpha$-Hölder continuous for all $\alpha \in(0,1)$, and on the other hand there exists a $\mathbb{N}$-valued function $\mathcal{N}(r)(r>0)$ such that $\lim _{r \rightarrow 0^{+}} \log \mathcal{N}(r) /|\log r|=0$ and for any open ball $B$ of radius $r>0$ in $\operatorname{conc}\left(G_{\mu}\right), \operatorname{conc}^{-1}(B)$ can be covered by at most $\mathcal{N}(r)$ balls of radius $r$. Thus conc preserves the Hausdorff dimension.

5.3. Proof of Theorem 1.4. (1) We prove that the following assertions are equivalent:

(i) $\alpha \in L_{\boldsymbol{\Phi}}$;

(ii) $E_{\left\{\boldsymbol{\Phi}^{(j)}\right\}, \mathbf{c}}(\alpha) \neq \emptyset$;

(iii) inf $\left\{P^{\mathbf{a}}\left(T_{1}, \mathbf{q} \cdot \mathbf{\Phi}\right)-\alpha \cdot \mathbf{q}: \mathbf{q} \in \mathbb{R}^{d}\right\} \geq 0$;

(iv) $\inf \left\{P^{\mathbf{a}}\left(T_{1}, \mathbf{q} \cdot \mathbf{\Phi}\right)-\alpha \cdot \mathbf{q}: \mathbf{q} \in \mathbb{R}^{d}\right\}>-\infty$;

For $\alpha \in L_{\boldsymbol{\Phi}}$ let $f_{\boldsymbol{\Phi}}(\alpha)=\max \left\{h_{\mu}^{\mathbf{a}}\left(T_{1}\right): \mu \in \mathcal{M}\left(X_{1}, T_{1}\right), \boldsymbol{\Phi}_{*}(\mu)=\alpha\right\}$. Since the mapping $\mu \in \mathcal{M}\left(X_{1}, T_{1}\right) \mapsto h_{\mu}^{\mathbf{a}}\left(T_{1}\right)$ is upper semi-continuous and affine, the equality $f_{\boldsymbol{\Phi}}(\alpha)=\inf \left\{P^{\mathbf{a}}\left(T_{1}, \mathbf{q} \cdot \boldsymbol{\Phi}\right)-\alpha \cdot \mathbf{q}: \mathbf{q} \in \mathbb{R}^{d}\right\}$ for $\alpha \in L_{\boldsymbol{\Phi}}$ is obtained by exactly the same arguments as those used to prove Theorem 5.2(iii) in [23]; one just replaces the usual entropy by the a-weighted one. Similarly, the proof of the equivalence 
between (i), (iii) and (iv) follow the same lines as that of Theorem 5.2 (ii) in [23]. The equivalence of (ii) and the other assertions will follow from the proof of Theorem 1.4(2) below.

(2) We only need to show

(5.19) $E_{\left\{\boldsymbol{\Phi}_{j}\right\}, \mathbf{c}}(\alpha) \neq \emptyset$ and $\operatorname{dim}_{H} E_{\left\{\boldsymbol{\Phi}_{j}\right\}, \mathbf{c}}(\alpha) \geq f_{\boldsymbol{\Phi}}(\alpha)$ if $\alpha \in L_{\boldsymbol{\Phi}}$

(5.20) $\operatorname{dim}_{H} E_{\left\{\boldsymbol{\Phi}_{j}\right\}, \mathbf{c}}(\alpha) \leq \inf \left\{P^{\mathbf{a}}\left(T_{1}, \mathbf{q} \cdot \boldsymbol{\Phi}\right)-\alpha \cdot \mathbf{q}: \mathbf{q} \in \mathbb{R}^{d}\right\}$ if $E_{\left\{\boldsymbol{\Phi}_{j}\right\}, \mathbf{c}}(\alpha) \neq \emptyset$,

This yields the equivalence of (i) and (ii) above, as well as the value of $\operatorname{dim}_{H} E_{\left\{\boldsymbol{\Phi}_{j}\right\}, \mathbf{c}}(\alpha)$.

Assertion (5.19) is an immediate consequence of Theorem 1.3 and the following lemma.

LEMMA 5.5. Let $\alpha=\left(\alpha_{1}, \ldots, \alpha_{d}\right) \in L_{\boldsymbol{\Phi}}$ and $\mu \in \mathcal{M}\left(X_{1}, T_{1}\right)$ such that $\int \mathbf{\Phi} d \mu=$ $\alpha$. We have $G_{\mu}\left(X_{1}, T_{1}\right) \subset E_{\left\{\mathbf{\Phi}_{j}\right\}, \mathbf{c}}(\alpha)$.

Proof of Lemma 5.5. Write each $\boldsymbol{\Phi}_{\mathbf{j}}$ as $\left(\varphi_{j, 1}, \ldots, \varphi_{j, d}\right)$. By definition of $\boldsymbol{\Phi}$, we have $\alpha_{i}=\sum_{j=1}^{r} \int \varphi_{j, i} d \mu$ for each $1 \leq i \leq d$. Moreover, by the definition of $G_{\mu}\left(X_{1}, T_{1}\right)$, we have $G_{\mu}\left(X_{1}, T_{1}\right) \subset E_{\varphi_{j, i}}\left(\int \varphi_{j, i} d \mu\right)$ for each $1 \leq j \leq r$ and $1 \leq i \leq d$, hence for each $x \in G_{\mu}\left(X_{1}, T_{1}\right)$ we have $\lim _{n \rightarrow \infty} \sum_{j=1}^{r} \frac{S_{\left\lfloor c_{j} n\right\rfloor} \varphi_{j, i}(x)}{\left\lfloor c_{j} n\right\rfloor}=\alpha_{i}$ for each $1 \leq i \leq d$. This yields $G_{\mu}\left(X_{1}, T_{1}\right) \subset E_{\left\{\boldsymbol{\Phi}_{j}\right\}, \mathbf{c}}(\alpha)$.

Now we establish (5.20). We define the following sequence of functions

$$
\boldsymbol{\Phi}_{\mathbf{c}, n}=n \sum_{j=1}^{r} \frac{S_{\left\lfloor c_{j} n\right\rfloor} \boldsymbol{\Phi}_{j}}{\left\lfloor c_{j} n\right\rfloor} .
$$

We first treat the case where $\left(X_{1}, T_{1}\right)$ is a full shift .

The upper bound in the full shift case. In this case we have the following lemma, which yields (5.20).

Lemma 5.6. Assume that $\left(X_{1}, T_{1}\right)$ is a full shift. Fix $\alpha \in \mathbb{R}^{d}$ and suppose that $E_{\left\{\boldsymbol{\Phi}_{j}\right\}, \mathbf{c}}(\alpha) \neq \emptyset$. For every $\varepsilon>0$ and $\mathbf{q} \in \mathbb{R}^{d}$, we have

$$
\operatorname{dim}_{H} E_{\left\{\Phi_{j}\right\}, \mathbf{c}}(\alpha, \varepsilon) \leq P^{\mathbf{a}}\left(T_{1}, \mathbf{q} \cdot \mathbf{\Phi}\right)-\alpha \cdot \mathbf{q}+\left(4|\mathbf{q}|+a_{1}\right) \varepsilon,
$$

where $E_{\left\{\boldsymbol{\Phi}_{j}\right\}, \mathbf{c}}(\alpha, \varepsilon)=\left\{x \in X_{1}: \limsup _{n \rightarrow \infty}\left|\boldsymbol{\Phi}_{\mathbf{c}, n}(x) / n-\alpha\right| \leq \varepsilon\right\}$. Consequently, if $E_{\left\{\boldsymbol{\Phi}_{j}\right\}, \mathbf{c}}(\alpha) \neq \emptyset$, then $\operatorname{dim}_{H} E_{\left\{\boldsymbol{\Phi}_{j}\right\}, \mathbf{c}}(\alpha) \leq \inf _{\mathbf{q} \in \mathbb{R}^{d}} P^{\mathbf{a}}\left(T_{1}, \mathbf{q} \cdot \boldsymbol{\Phi}\right)-\alpha \cdot \mathbf{q}$, i.e., (5.20) holds.

Proof of Lemma 5.6. Fix $\varepsilon>0$ and $\mathbf{q} \in \mathbb{R}^{d}$. For each $1 \leq j \leq r$, choose a continuous function $\widetilde{\boldsymbol{\Phi}}_{j}=\left(\widetilde{\varphi}_{j, 1}, \ldots, \widetilde{\varphi}_{j, d}\right)$ in $C\left(X_{1}\right)^{d}$ such that each $\widetilde{\varphi}_{j, d}$ is Hölder continuous and

$$
\sup _{1 \leq i \leq d}\left\|\widetilde{\varphi}_{j, d}-\varphi_{j, d}\right\|_{\infty} \leq \varepsilon / r
$$

Then we define $\widetilde{\boldsymbol{\Phi}}=\sum_{j=1}^{r} \widetilde{\boldsymbol{\Phi}}_{j}$, and the sequence of functions

$$
\widetilde{\boldsymbol{\Phi}}_{\mathbf{c}, n}=n \sum_{j=1}^{r} \frac{S_{\left\lfloor c_{j} n\right\rfloor} \widetilde{\boldsymbol{\Phi}}_{j}}{\left\lfloor c_{j} n\right\rfloor} \quad(n \geq 1) .
$$


Endow the space $\mathbb{R}^{d}$ with the norm $\left|\left(z_{1}, \ldots, z_{d}\right)\right|=\max _{1 \leq i \leq d}\left|z_{i}\right|$. By construction we have $\lim \sup _{n \rightarrow \infty}\left\|\widetilde{\boldsymbol{\Phi}}_{\mathbf{c}, n}-\boldsymbol{\Phi}_{\mathbf{c}, n}\right\|_{\infty} / n \leq \varepsilon$ so

$$
E_{\left\{\boldsymbol{\Phi}_{j}\right\}, \mathbf{c}}(\alpha, \varepsilon) \subset E_{\left\{\widetilde{\boldsymbol{\Phi}}_{j}\right\}, \mathbf{c}}(\alpha, 2 \varepsilon)=\left\{x \in X_{1}: \limsup _{n \rightarrow \infty}\left|\widetilde{\boldsymbol{\Phi}}_{\mathbf{c}, n}(x) / n-\alpha\right| \leq 2 \varepsilon\right\} .
$$

The definition of the a-weighted topological pressure implies

$$
\left|P^{\mathbf{a}}\left(T_{1}, \mathbf{q} \cdot \widetilde{\mathbf{\Phi}}\right)-P^{\mathbf{a}}\left(T_{1}, \mathbf{q} \cdot \mathbf{\Phi}\right)\right| \leq|\mathbf{q}| \varepsilon .
$$

Let us denote by $\mu_{\mathbf{q}}$ the unique a-weighted equilibrium state of $\mathbf{q} \cdot \widetilde{\mathbf{\Phi}}$ (see Theorem 3.5). The following key property holds.

Lemma 5.7. Let $\mathbf{q} \in \mathbb{R}^{d}$. For all $x \in X_{1}$, we have $\limsup _{n \rightarrow \infty} f_{n}(x)^{1 / n} \geq 1$, where

$$
f_{n}(x)=\frac{\mu_{\mathbf{q}}\left(B\left(x, e^{-n / a_{1}}\right)\right)}{\exp \left(\left(\mathbf{q} \cdot \widetilde{\mathbf{\Phi}}_{\mathbf{c}, n}(x)-n P^{\mathbf{a}}\left(T_{1}, \mathbf{q} \cdot \widetilde{\mathbf{\Phi}}\right)\right) / a_{1}\right)} .
$$

It is worth mentioning that the idea of considering the asymptotic behavior of such a function $f_{n}$ at each point of $X_{1}$ goes back to [35] for the upper bound estimate of $\operatorname{dim}_{H} X_{1}$ when $k=2$. The proof of Lemma 5.7 will be given later. To finish the proof of Lemma 5.6, we need the following classical lemma.

LEMMA 5.8 ([8], Ch. 14). Let $E$ be a non-empty subset of a compact metric space $(Y, d)$ endowed with an ultrametric distance. Let $\nu$ be a positive Borel measure on $Y$. Then $\operatorname{dim}_{H} E \leq \sup _{x \in E} \liminf _{r \rightarrow 0^{+}} \frac{\log \nu(B(x, r))}{\log r}$.

Now, if $x \in E_{\left\{\widetilde{\boldsymbol{\Phi}}_{j}\right\}, \mathbf{c}}(\alpha, 2 \varepsilon)$ then, due to Lemma 5.7, for infinitely many $n$ we have simultaneously $f_{n}(x) \geq \exp (-n \varepsilon)$, and $\exp \left(\mathbf{q} \cdot \widetilde{\mathbf{\Phi}}_{\mathbf{c}, n}(x)\right) \geq \exp (n \alpha \cdot \mathbf{q})-3|\mathbf{q}| \varepsilon n$. Consequently,

$$
\liminf _{n \rightarrow \infty} \frac{\log \mu_{\mathbf{q}}\left(B\left(x, e^{-n / a_{1}}\right)\right)}{-n / a_{1}} \leq P^{\mathbf{a}}\left(T_{1}, \mathbf{q} \cdot \widetilde{\mathbf{\Phi}}\right)-\alpha \cdot \mathbf{q}+\left(3|\mathbf{q}|+a_{1}\right) \varepsilon .
$$

Now, Lemma 5.8 and (5.22) yield

$$
\begin{aligned}
\operatorname{dim}_{H} E_{\left\{\boldsymbol{\Phi}_{j}\right\}, \mathbf{c}}(\alpha, \varepsilon) & \leq \operatorname{dim}_{H} E_{\left\{\widetilde{\boldsymbol{\Phi}}_{j}\right\}, \mathbf{c}}(\alpha, 2 \varepsilon) \leq P^{\mathbf{a}}\left(T_{1}, \mathbf{q} \cdot \widetilde{\boldsymbol{\Phi}}\right)-\alpha \cdot \mathbf{q}+\left(3|\mathbf{q}|+a_{1}\right) \varepsilon \\
& \leq P^{\mathbf{a}}\left(T_{1}, \mathbf{q} \cdot \boldsymbol{\Phi}\right)-\alpha \cdot \mathbf{q}+\left(4|\mathbf{q}|+a_{1}\right) \varepsilon .
\end{aligned}
$$

Letting $\varepsilon \rightarrow 0$, we obtain $\operatorname{dim}_{H} E_{\left\{\boldsymbol{\Phi}_{j}\right\}, \mathbf{c}}(\alpha) \leq P^{\mathbf{a}}\left(T_{1}, \mathbf{q} \cdot \boldsymbol{\Phi}\right)-\alpha \cdot \mathbf{q}$. Since $\mathbf{q} \in \mathbb{R}^{d}$ is arbitrarily given, we have

$$
\operatorname{dim}_{H} E_{\left\{\boldsymbol{\Phi}_{j}\right\}, \mathbf{c}}(\alpha) \leq \inf _{\mathbf{q} \in \mathbb{R}^{d}} P^{\mathbf{a}}\left(T_{1}, \mathbf{q} \cdot \mathbf{\Phi}\right)-\alpha \cdot \mathbf{q} .
$$

This finishes the proof of Lemma 5.6.

Before we prove Lemma 5.7, we give some auxiliary lemmas.

Lemma 5.9 ([28], Lemma 4.1). Let $m \geq 1$ be an integer. For $1 \leq j \leq m$ let $f_{j}: \mathbb{N} \rightarrow \mathbb{R}, \beta_{j}>0$ and $\lambda_{j}>0$. If $\sup _{n \geq 1}\left|f_{j}(n+1)-f_{j}(n)\right|<\infty$ for each $j$, then

$$
\limsup _{t \rightarrow \infty} \frac{1}{t} \sum_{j=1}^{m}\left(\beta_{j} f_{j}\left(\left\lfloor\frac{t}{\lambda_{j}}\right\rfloor\right)-f_{j}\left(\left\lfloor\frac{\beta_{j} t}{\lambda_{j}}\right\rfloor\right)\right) \geq 0 .
$$


Proof of Lemma 5.7. Fix $\mathbf{q} \in \mathbb{R}^{d}$ and $x \in X_{1}$. By Lemma 5.2, we have

$\mu_{\mathbf{q}}\left(B\left(x, e^{-n / a_{1}}\right)\right) \approx \exp \left(\frac{-n P^{\mathbf{a}}\left(T_{1}, \mathbf{q} \cdot \widetilde{\mathbf{\Phi}}\right)+S_{n}(\mathbf{q} \cdot \widetilde{\mathbf{\Phi}})(x)}{a_{1}}\right) \prod_{i=1}^{k-1} \frac{\widetilde{\phi}^{(i)}\left(\tau_{i}\left(x_{\mid \ell_{i+1}(n)}\right)\right)^{1 / A_{i+1}}}{\widetilde{\phi}^{(i)}\left(\tau_{i}\left(x_{\mid \ell_{i}(n)}\right)\right)^{1 / A_{i}}}$

for certain functions $\widetilde{\phi}^{(i)}$ on $\mathcal{A}_{i}^{*}(1 \leq i \leq k)$. Combining this with the definition of $f_{n}(x)$ yields

$$
f_{n}(x) \approx \exp \left(\frac{S_{n}(\mathbf{q} \cdot \widetilde{\boldsymbol{\Phi}})(x)-\widetilde{\boldsymbol{\Phi}}_{\mathbf{c}, n}(x)}{a_{1}}\right) \prod_{i=1}^{k-1} \frac{\widetilde{\phi}^{(i)}\left(\tau_{i}\left(x_{\mid \ell_{i+1}(n)}\right)\right)^{1 / A_{i+1}}}{\widetilde{\phi}^{(i)}\left(\tau_{i}\left(x_{\mid \ell_{i}(n)}\right)\right)^{1 / A_{i}}} .
$$

Notice that $\widetilde{\mathbf{\Phi}}_{\mathbf{c}, n}$ has the following form (cf. (5.21)):

$$
\widetilde{\mathbf{\Phi}}_{\mathbf{c}, n}=S_{n}(\mathbf{q} \cdot \widetilde{\mathbf{\Phi}})+\sum_{j=1}^{r}\left(\frac{n S_{\left\lfloor c_{j} n\right\rfloor}\left(\mathbf{q} \cdot \widetilde{\boldsymbol{\Phi}}_{j}\right)}{\left\lfloor c_{j} n\right\rfloor}-S_{n}\left(\mathbf{q} \cdot \widetilde{\boldsymbol{\Phi}}_{j}\right)\right) .
$$

Now, for $n \geq 1$, let us define

$$
\begin{cases}u^{(j)}(n)=a_{1}^{-1} S_{n} \widetilde{\Phi}_{j}(x) & \text { for } 1 \leq j \leq r \\ \widetilde{u}^{(i)}(n)=a_{1}^{-1} \log \widetilde{\phi}^{(i)}\left(\tau_{i}\left(x_{\mid n}\right)\right) & \text { for } 1 \leq i \leq k-1 .\end{cases}
$$

Since the potentials $\widetilde{\boldsymbol{\Phi}}$ and $\widetilde{\boldsymbol{\Phi}}_{j}$ are Hölder continuous, for any $v \in\left\{u^{(j)}, \widetilde{u}^{(i)}: 1 \leq j \leq\right.$ $r, 1 \leq i \leq k-1\}$ the sequence $(v(n))_{n \geq 1}$ satisfies $v(n+1)-v(n)=O(1)$. Then, by using (5.23) we can get

$$
\frac{\log f_{n}(x)}{n}=\frac{1}{n}\left(\sum_{j=1}^{r}\left(u^{(j)}(n)-\frac{1}{c_{j}} u^{(j)}\left(\left\lfloor c_{j} n\right\rfloor\right)\right)+\sum_{i=1}^{k-1}\left(\frac{\widetilde{u}^{(i)}\left(\left\lfloor\widetilde{c}_{i+1} n\right\rfloor\right)}{\widetilde{c}_{i+1}}-\frac{\widetilde{u}^{(i)}\left(\left[\widetilde{c}_{i} n\right\rfloor\right)}{\widetilde{c}_{i}}\right)+O(1)\right),
$$

where $\widetilde{c}_{i}:=A_{i} / a_{1}$ for $i=1, \ldots, k$. Then, the fact that $\lim \sup _{n \rightarrow \infty} \frac{\log f_{n}(x)}{n} \geq 0$ comes from Lemma 5.9. This finishes the proof of Lemma 5.7.

The upper bound in the general case. We show that the upper bound for $\operatorname{dim}_{H} E_{\left\{\boldsymbol{\Phi}_{j}\right\}, \mathbf{c}}(\alpha)$ is valid without any assumption like specification on $\left(X_{1}, T_{1}\right)$.

For each $p \geq 1$, let $\Gamma_{p}$ be the natural injection of $\left(X_{1}, d_{\mathbf{a}}\right)$ into $\left(\mathcal{L}_{p}^{\mathbb{N}}, d_{\mathbf{a} / p}\right)$. The map $\Gamma_{p}$ is easily seen to be a bi-Lipschitz map from $X_{1}$ onto its image, so it preserves Hausdorff dimension.

For each $1 \leq j \leq r$, the $\mathbb{R}^{d}$ valued continuous potentials $\boldsymbol{\Phi}_{j}$ can be extended to an $\mathbb{R}^{d}$ valued continuous potentials $\widehat{\boldsymbol{\Phi}}_{j}$ defined on $\left(\mathcal{A}_{1}^{\mathbb{N}}, d_{\mathbf{a}}\right)$. By construction, for each $\alpha \in L_{\boldsymbol{\Phi}}$, we have $\Gamma_{p}\left(E_{\left\{\boldsymbol{\Phi}_{j}\right\}, \mathbf{c}}(\alpha)\right) \subset E_{\left\{S_{p} \widehat{\boldsymbol{\Phi}}_{j} / p\right\}, \mathbf{c}}(\alpha)$ so the above study applied to the full shift $\left(\mathcal{L}_{p}^{\mathbb{N}}, T_{1}^{p}, d_{\mathbf{a} / p}\right)$ yields

$$
\operatorname{dim}_{H} E_{\left\{\boldsymbol{\Phi}_{j}\right\}, \mathbf{c}}(\alpha) \leq \max \left\{h_{\mu}^{\mathbf{a} / p}\left(T_{1}^{p}\right): \mu \in \mathcal{M}\left(\mathcal{L}_{p}^{\mathbb{N}}, T_{1}^{p}\right), \int S_{p} \widehat{\boldsymbol{\Phi}} / p d \mu=\alpha\right\} .
$$

Let $\nu_{p}$ be one measure realizing the above maximum and extend it as an invariant measure on $\left(\mathcal{A}_{1}^{\mathbb{N}}, T_{1}^{p}\right)$ (notice that $\nu_{p}$ is supported by $\mathcal{L}_{p}^{\mathbb{N}}$ ). Then define on $\mathcal{A}_{1}^{\mathbb{N}}$ the $T_{1}$-invariant measure $\mu_{p}=\frac{1}{p} \sum_{j=0}^{p-1} \nu_{p} \circ T_{1}^{-j}$. By the affinity of $h_{(\cdot)}^{\mathbf{a} / p}\left(T_{1}^{p}\right)$ we have $h_{\mu_{p}}^{\mathbf{a} / p}\left(T_{1}^{p}\right)=h_{\nu_{p}}^{\mathbf{a} / p}\left(T_{1}^{p}\right)$ since all the measures $\nu_{p} \circ T_{1}^{-j}$ have the same $\mathbf{a} / p$-weighted 
entropy with respect to $T_{1}^{p}$. Consequently, $h_{\nu_{p}}^{\mathbf{a} / p}\left(T_{1}^{p}\right)=h_{\mu_{p}}^{\mathbf{a} / p}\left(T_{1}^{p}\right)=p h_{\mu_{p}}^{\mathbf{a} / p}\left(T_{1}\right)=$ $h_{\mu_{p}}^{\mathbf{a}}\left(T_{1}\right)$. Finally, by construction,

$$
\operatorname{dim}_{H} E_{\left\{\boldsymbol{\Phi}_{j}\right\}, \mathbf{c}}(\alpha) \leq h_{\mu_{p}}^{\mathbf{a}}\left(T_{1}\right) \text { and } \int \widehat{\boldsymbol{\Phi}} d \mu_{p}=\alpha
$$

Now, without loss of generality we can suppose that $\mu_{p}$ converges to an element $\mu \in$ $\mathcal{M}\left(\mathcal{A}_{1}^{\mathbb{N}}, T_{1}\right)$ in the weak-star topology. We have both $h_{\mu}^{\mathbf{a}}\left(T_{1}\right) \geq \lim \sup _{p \rightarrow \infty} h_{\mu_{p}}^{\mathbf{a}}\left(T_{1}\right)$ and $\int \boldsymbol{\Phi} d \mu=\alpha$. Assume that $\mu$ is supported on $X_{1}$. Then by (5.24) we have $\operatorname{dim}_{H} E_{\left\{\boldsymbol{\Phi}_{j}\right\}, \mathbf{c}}(\alpha) \leq \max \left\{h_{\mu}^{\mathbf{a}}\left(T_{1}\right): \mu \in \mathcal{M}\left(X_{1}, T_{1}\right), \int \boldsymbol{\Phi} d \mu=\alpha\right\}$ as desired. Now, the fact that $\mu$ is supported on $X_{1}$ follows from the same argument as that used in the proof of [28, Theorem 1.1] to build an invariant measure of full Hausdorff dimension on $X_{1}$.

(3) We will use a modification of the Moran construction achieved in the proof of Theorem 5.3. To do so we need some preparation.

Let $\nu_{1}$ be an invariant measure on $X_{1}$ such that $h_{\nu_{1}}^{\mathbf{a}}\left(T_{1}\right)=\operatorname{dim}_{H} X_{1}$ (the existence of such a measure was first proved in [28]; this fact is also a consequence of Theorem 1.4 applied to the null potential). Fix $\varepsilon>0$, and for each $1 \leq i \leq k$ let $h_{i}=h_{\nu_{1} \circ \tau_{i-1}^{-1}}\left(T_{i}\right)-$ $\varepsilon /\left(a_{1}+\cdots+a_{k}\right)$. By our assumption, $L_{\boldsymbol{\Phi}}$ is not a singleton. Let $\nu \in \mathcal{M}\left(X_{1}, T_{1}\right)$ such that $\nu(\boldsymbol{\Phi}) \neq \nu_{1}(\boldsymbol{\Phi})$. Then take a large positive integer $n$ so that

$$
h_{\nu_{2} \circ \tau_{i-1}^{-1}}\left(T_{i}\right) \geq h_{\nu_{1} \circ \tau_{i-1}^{-1}}\left(T_{i}\right)-\varepsilon /\left(a_{1}+\cdots+a_{k}\right), \quad(1 \leq i \leq k)
$$

where $\nu_{2}=(1-1 / n) \nu_{1}+(1 / n) \nu$. Note that $\delta=\left|\nu_{2}(\mathbf{\Phi})-\nu_{1}(\mathbf{\Phi})\right|>0$.

By Remark 4.4, for each $l \in\{1,2\}$ there exists a sequence $\left(\mu_{l, p}\right)_{p=1}^{\infty}$ of quasi Bernoulli measures such that $\mu_{p} \in \mathcal{M}\left(\mathcal{L}_{p}^{\mathbb{N}}, T_{1}^{p}\right)$ and

$$
h_{\mu_{l, p} \circ \tau_{i-1}^{-1}}\left(T_{i}^{p}\right) \geq p\left(h_{\nu_{l} \circ \tau_{i-1}^{-1}}\left(T_{i}\right)-\varepsilon /\left(a_{1}+\cdots+a_{k}\right)\right)-1 /(2 p)=p h_{i}-1 /(2 p)
$$

for each $1 \leq i \leq k$ and for $p \geq 1$.

For each $1 \leq j \leq r$ and $1 \leq i \leq d$, take $\widetilde{\varphi}_{j, i} \in C\left(\mathcal{A}_{1}^{\mathbb{N}}\right)$ such that $\widetilde{\varphi}_{j, i}$ is Hölder continuous and $\left\|\widetilde{\varphi}_{j, i}-\varphi_{j, i}\right\|_{\infty} \leq \delta / 8 r$. Moreover, for each $l \in\{1,2\}$ the sequence $\left(\mu_{l, p}\right)_{p=1}^{\infty}$ can be chosen so that $\left|\mu_{l, p}\left(\widetilde{\varphi}_{j, i}\right)-\nu_{l}\left(\widetilde{\varphi}_{j, i}\right)\right| \leq \delta / 8 r$.

We are going to construct a sequence $\left(N_{p}^{\prime}\right)_{p=1}^{\infty}$ such that the set

$$
D=\bigcap_{l=1}^{2}\left\{x \in G=\prod_{p=1}^{\infty} \mathcal{L}_{p}^{N_{p}^{\prime}}: \liminf _{n \rightarrow \infty}\left|\boldsymbol{\Phi}_{\mathbf{c}, n}(x)-n \nu_{l}(\mathbf{\Phi})\right| / n \leq \delta / 4\right\},
$$

which is a subset of $\mathcal{A}_{1}^{\mathbb{N}} \backslash \bigcup_{\alpha \in L_{\boldsymbol{\Phi}}} E_{\left\{\boldsymbol{\Phi}_{j}\right\}, \mathbf{c}}(\alpha)$, contains a Moran subset $G_{\mu}$ with $\operatorname{dim}_{H} G_{\mu} \geq \operatorname{dim}_{H} X_{1}-2 \varepsilon$. Then, by using the same imbedding conc as in the proof of Theorem 5.3, we will get $\operatorname{conc}\left(G_{\mu}\right) \subset X_{1} \backslash \bigcup_{\alpha \in L_{\boldsymbol{\Phi}}} E_{\left\{\boldsymbol{\Phi}_{j}\right\}, \mathbf{c}}(\alpha)$ and $\operatorname{dim}_{H} \operatorname{conc}\left(G_{\mu}\right) \geq$ $\operatorname{dim}_{H} X_{1}-\varepsilon$.

Now we briefly explain how to modify the Moran construction done in the proof of Theorem 5.3 to build $G$ and $G_{\mu}$. At first, without loss of generality, we suppose that the $c_{j}$ 's are greater than 1 . Also, we include the potentials $\widetilde{\varphi}_{j, i}$ in the family $\widetilde{\mathcal{C}}$. Then, the only changes are that for each $p \geq 1$, one takes $\mu_{2 p-1}=\nu_{1, p}$ and $\mu_{2 p}=\nu_{2, p}$. Then, for $p \geq 1$, let $n_{p}=\sum_{i=1}^{p-1} i N_{i}^{\prime}+\sqrt{p N_{p}^{\prime}}$. For $p$ large enough, for each $1 \leq j \leq r$ we have

$$
\left\lfloor c_{j} n_{p}\right\rfloor \in\left[\sum_{i=1}^{p-1} i N_{i}^{\prime}+\sqrt{p N_{p}^{\prime}}, \sum_{i=1}^{p} i N_{i}^{\prime}\right]
$$


so that for each $x \in G_{\mu}, 1 \leq j \leq r$ and $1 \leq i \leq d$ we have

$$
\limsup _{p \rightarrow \infty}\left|S_{\left\lfloor c_{j} n_{2 p-1}\right\rfloor} \widetilde{\varphi}_{j, i}(x) /\left\lfloor c_{j} n_{2 p-1}\right\rfloor-\nu_{1}\left(\widetilde{\varphi}_{j, i}\right)\right| \leq \delta / 8 r
$$

and

$$
\limsup _{p \rightarrow \infty}\left|S_{\left\lfloor c_{j} n_{2 p}\right\rfloor} \widetilde{\varphi}_{j, i}(x) /\left\lfloor c_{j} n_{2 p}\right\rfloor-\nu_{2}\left(\widetilde{\varphi}_{j, i}\right)\right| \leq \delta / 8 r .
$$

Consequently, by construction for each $x \in G_{\mu}$, we have

$$
\limsup _{p \rightarrow \infty}\left|\boldsymbol{\Phi}_{\mathbf{c}, n_{2 p-1}}(x) / n_{2 p-1}-\nu_{1}(\boldsymbol{\Phi})\right| \leq \delta / 4 \text { and } \lim _{p \rightarrow \infty}\left|\boldsymbol{\Phi}_{\mathbf{c}, n_{2 p}}(x) / n_{2 p}-\nu_{2}(\boldsymbol{\Phi})\right| \leq \delta / 4,
$$

so $G_{\mu} \subset D$. Moreover, the simultaneous controls from below of the entropies $h_{\nu_{l, p} \circ \tau_{i-1}^{-1}}\left(T_{i}^{p}\right)$ by the $p h_{i}-1 /(2 p)$ yield, for every $x \in G_{\mu}$,

$$
\liminf _{n \rightarrow \infty} \frac{\log \nu\left(B\left(x, e^{-n / a_{1}}\right)\right)}{-n / a_{1}} \geq \sum_{i=1}^{k} a_{i} h_{i} \geq \operatorname{dim}_{H} X_{1}-\varepsilon
$$

REMARK 5.10. Without assuming the specification property, for any $\mu \in$ $\mathcal{M}\left(X_{1}, T_{1}\right)$, we have $\operatorname{dim}_{P} G_{\mu}\left(X_{1}, T_{1}\right) \leq h_{\mu}^{\mathbf{a}}\left(T_{1}\right)$, where $\operatorname{dim}_{P}$ denotes the packing dimension (cf. [34]). This is done by using a recent result of Reeves [41]. To see it, let $\left(\phi_{n}\right)_{n=1}^{\infty}$ be a family of continuous functions on $X_{1}$ which is dense in $C\left(X_{1}\right)$. For $m \geq 1$, define $\boldsymbol{\Phi}_{m}=\left(\phi_{1}, \ldots, \phi_{m}\right)$ and

$$
E_{m}=\left\{x \in X_{1}: \lim _{n \rightarrow \infty} \frac{1}{n} S_{n} \mathbf{\Phi}_{m}(x)=\left(\int \phi_{1} d \mu, \ldots, \int \phi_{m} d \mu\right)\right\}
$$

By Reeves' result [41],

$$
\operatorname{dim}_{P} E_{m} \leq \sum_{i=1}^{k} \sup \left\{a_{i} h_{\eta \circ \pi^{-1}}\left(T_{i}\right): \int \phi_{j} d \eta=\int \phi_{j} d \mu \text { for } 1 \leq j \leq m\right\} .
$$

(Indeed, Reeves only proved the above result in the case $k=2$ and $m=1$ in the full shift case with the equality rather that $\leq$; however, it can be extended to the above general form without additional difficulty.) Since $\left(\phi_{n}\right)_{n=1}^{\infty}$ is dense in $C\left(X_{1}\right)$, by using the upper semi-continuity of the entropy function, we obtain

$$
\limsup _{n \rightarrow \infty} \operatorname{dim}_{P} E_{m} \leq \sum_{i=1}^{k} a_{i} h_{\mu \circ \pi^{-1}}\left(T_{i}\right)=h_{\mu}^{\mathbf{a}}\left(T_{1}\right) .
$$

Since $G_{\mu}\left(X_{1}, T_{1}\right) \subseteq E_{m}$ for each $m$, we obtain the upper bound $\operatorname{dim}_{P} G_{\mu}\left(X_{1}, T_{1}\right) \leq$ $h_{\mu}^{\mathbf{a}}\left(T_{1}\right)$. 
6. Multifractal analysis of asymptotically additive potentials and application to weighted equilibrium states. For $\boldsymbol{\Phi}=\left(\Phi_{1}, \ldots, \Phi_{d}\right) \in \mathcal{C}_{\text {asa }}\left(X_{1}, T_{1}\right)^{d}$, where $\Phi_{i}=\left(\log \phi_{n, i}\right)_{n=1}^{\infty}=:\left(\Phi_{n, i}\right)_{n=1}^{\infty}$, and $\mu \in \mathcal{M}\left(X_{1}, T_{1}\right)$, write $\boldsymbol{\Phi}_{*}(\mu)=$ $\left(\left(\Phi_{1}\right)_{*}(\mu), \ldots,\left(\Phi_{d}\right)_{*}(\mu)\right)$ and define $L_{\boldsymbol{\Phi}}=\left\{\boldsymbol{\Phi}_{*}(\mu): \mu \in \mathcal{M}\left(X_{1}, T_{1}\right)\right\}$.

Let $\left\{\boldsymbol{\Phi}^{(j)}\right\}_{1 \leq j \leq r}$ be a family of elements of $\mathcal{C}_{a s a}\left(X_{1}, T_{1}\right)^{d}$. Let $\mathbf{c}=\left(c_{1}, \ldots, c_{r}\right)$ be a real vector with positive entries. For $\alpha \in \mathbb{R}^{d}$, define

$$
E_{\left\{\boldsymbol{\Phi}^{(j)}\right\}, \mathbf{c}}(\alpha)=\left\{x \in X_{1}: \lim _{n \rightarrow \infty} \sum_{j=1}^{r} \frac{\boldsymbol{\Phi}_{\left\lfloor c_{j} n\right\rfloor}^{(j)}}{\left\lfloor c_{j} n\right\rfloor}(x)=\alpha\right\} .
$$

Theorem 1.4 has the following easy extension, which is obtained thanks to the density of continuous additive potentials in $\mathcal{C}_{a s a}\left(X_{1}, T_{1}\right)^{d}$. This extension is useful to get results on the multifractal analysis of weighted equilibrium states.

Theorem 6.1. Let $\boldsymbol{\Phi}=\sum_{j=1}^{r} \boldsymbol{\Phi}^{(j)}$.

1. For $\alpha \in \mathbb{R}^{d}, E_{\left\{\boldsymbol{\Phi}^{(j)}\right\}, \mathbf{c}}(\alpha) \neq \emptyset$ if and only if $\alpha \in L_{\boldsymbol{\Phi}}$.

2. For $\alpha \in L_{\boldsymbol{\Phi}}$, we have

$$
\begin{aligned}
\operatorname{dim}_{H} E_{\left\{\boldsymbol{\Phi}^{(j)}\right\}, \mathbf{c}}(\alpha) & =\max \left\{h_{\mu}^{\mathbf{a}}\left(T_{1}\right): \mu \in \mathcal{M}\left(X_{1}, T_{1}\right), \mathbf{\Phi}_{*}(\mu)=\alpha\right\} \\
& =\inf \left\{P^{\mathbf{a}}\left(T_{1}, \mathbf{q} \cdot \mathbf{\Phi}\right)-\alpha \cdot \mathbf{q}: \mathbf{q} \in \mathbb{R}^{d}\right\} .
\end{aligned}
$$

3. Suppose that $L_{\boldsymbol{\Phi}}$ is not a singleton. Then the set $X \backslash \bigcup_{\alpha \in L_{\boldsymbol{\Phi}}} E_{\left\{\Phi^{(j)}\right\}, \mathbf{c}}(\alpha)$ is of full Hausdorff dimension.

Example 6.2. Generally, the level sets $E_{\left\{\boldsymbol{\Phi}^{(j)}\right\}, \mathbf{c}}(\alpha)$ depend on c. For example, let $X=\{0,1\}^{\mathbb{N}}$, and let $g \in C(X)$ be given by $g(x)=x_{1}$ for $x=\left(x_{i}\right)_{i=1}^{\infty} \in X$. Set $\Phi^{(1)}=\left(S_{n} g\right)_{n=1}^{\infty}$ and $\Phi^{(2)}=\left(-S_{n} g\right)_{n=1}^{\infty}$. Then $E_{\left\{\Phi^{(j)}\right\}_{j=1}^{2},(1,1)}(0)=X$, however $E_{\left\{\Phi^{(j)}\right\}_{j=1}^{2},(1,2)}(0) \neq X$ (it is easy to check that $x=0^{1} 1^{2} 0^{4} 1^{8} \cdots 0^{2^{2 n}} 1^{2^{2 n+1}} \cdots \notin$ $\left.E_{\left\{\boldsymbol{\Phi}^{(j)}\right\}_{j=1}^{2},(1,2)}(0)\right)$.

Application to the multifractal analysis of a-weighted Gibbs measures. In this section we suppose that $\left(X_{1}, T_{1}\right)$ is the full shift $\left(\mathcal{A}_{1}^{\mathbb{N}}, T_{1}\right)$.

Let $\Phi=\left(\log \phi_{n}\right)_{n=1}^{\infty} \in \mathcal{C}_{a a}\left(X_{1}, T_{1}\right)$ and suppose that $\Phi$ satisfies the bounded distortion property. Let $\mu$ be the a-weighted Gibbs measure $\mu$ associated with $\Phi$.

Due to the quasi-Bernoulli property of $\mu$, for each $1 \leq i \leq k$, the potential $\mathbf{\Psi}_{\mu}^{(i)}:=\left(\log \mu \circ \tau_{i-1}^{-1}\left(\tau_{i-1}\left(x_{\mid n}\right)\right)\right)_{n=1}^{\infty}$ belongs to $\mathcal{C}_{a a}\left(X_{1}, T_{1}\right)$.

We have the following result about the multifractal analysis of $\mu$.

Theorem 6.3. For $\alpha \in \mathbb{R}_{+}$let

$$
E_{\mu}(\alpha)=\left\{x \in X_{1}: \lim _{r \rightarrow 0^{+}} \frac{\log \mu(B(x, r))}{\log r}=\alpha\right\} .
$$

Let $\boldsymbol{\Psi}_{\mu}=\sum_{i=1}^{k} a_{i} \mathbf{\Psi}_{\mu}^{(i)}$. Let $L_{\mu}=L_{-\boldsymbol{\Psi}_{\mu}}=\left\{-\left(\boldsymbol{\Psi}_{\mu}\right)_{*}(\lambda): \lambda \in \mathcal{M}\left(X_{1}, T_{1}\right)\right\}$. Then, for all $\alpha \geq 0, E_{\mu}(\alpha) \neq \emptyset$ if and only if $\alpha \in L_{\mu}$. Moreover, for $\alpha \in L_{\mu}$ we have

$$
\begin{aligned}
\operatorname{dim}_{H} E_{\mu}(\alpha) & =\max \left\{h_{\lambda}^{\mathbf{a}}\left(T_{1}\right): \lambda \in \mathcal{M}\left(X_{1}, T_{1}\right),\left(\mathbf{\Psi}_{\mu}\right)_{*}(\lambda)=-\alpha\right\} \\
& =\inf \left\{P^{\mathbf{a}}\left(T_{1}, q \mathbf{\Psi}_{\mu}\right)+\alpha q: q \in \mathbb{R}\right\} .
\end{aligned}
$$


Proof. This result is just a corollary of Theorem 6.1. Indeed, thanks to (5.1) we can write

$$
\begin{aligned}
\frac{\log \mu\left(B\left(x, e^{-n / a_{1}}\right)\right)}{-n / a_{1}} & =-a_{1} \frac{\boldsymbol{\Psi}_{\mu, n}^{(1)}(x)}{n}-a_{1} \sum_{i=2}^{k} \frac{\boldsymbol{\Psi}_{\mu, \ell_{i}(n)}^{(i)}(x)}{n}-\frac{\boldsymbol{\Psi}_{\mu, \ell_{i-1}(n)}^{(i)}(x)}{n}+O(1 / n) \\
& =-a_{1} \frac{\boldsymbol{\Psi}_{\mu, n}^{(1)}(x)}{n}-a_{1} \sum_{i=2}^{k} \frac{b_{i} \boldsymbol{\Psi}_{\mu,\left\lfloor b_{i} n\right\rfloor}^{(i)}(x)}{\left\lfloor b_{i} n\right\rfloor}-\frac{b_{i-1} \boldsymbol{\Psi}_{\mu,\left\lfloor b_{i-1} n\right\rfloor}^{(i)}(x)}{\left\lfloor b_{i-1} n\right\rfloor}+O(1 / n),
\end{aligned}
$$

with $b_{i}=\left(a_{1}+\cdots+a_{i}\right) / a_{1}$. Thus, any set $E_{\mu}(\alpha)$ takes the form $E_{\left\{\boldsymbol{\Phi}^{(j)}\right\}, \mathbf{c}}(\alpha)$, with $\sum_{j=1}^{r} \boldsymbol{\Phi}^{(j)}=-\boldsymbol{\Psi}_{\mu}$. $\mathrm{u}$

More geometric applications. A parallelepiped is a subset of $X_{1}$ of the form

$$
R\left(I_{1}, \ldots, I_{k}\right)=\bigcap_{i=1}^{k} \tau_{i-1}^{-1}\left(I_{i}\right), \text { with } I_{i} \in \bigcup_{n \geq 0} \mathcal{A}_{i}^{n} .
$$

If we fix $0 \leq \lambda_{1} \leq \cdots \leq \lambda_{k}$ and set

$$
R_{n}\left(\lambda_{1}, \ldots, \lambda_{k}, x\right)=R\left(x_{\left\lfloor\left\lfloor\lambda_{1} n\right\rfloor\right.}, \ldots, \tau_{i-1}\left(x_{\mid\left\lfloor\lambda_{i} n\right\rfloor}\right), \cdots, \tau_{k-1}\left(x_{\left\lfloor\left\lfloor\lambda_{k} n\right\rfloor\right.}\right)\right),
$$

then

$$
\log \mu\left(R_{n}\left(\lambda_{1}, \ldots, \lambda_{k}, x\right)\right)=\sum_{i=1}^{k} \boldsymbol{\Psi}_{\mu,\left\lfloor\lambda_{i} n\right\rfloor}^{(i)}(x)-\boldsymbol{\Psi}_{\mu,\left\lfloor\lambda_{i-1} n\right\rfloor}^{(i)}(x)+O(1),
$$

with the convention $\lambda_{0}=0$. Consequently, Theorem 6.1 makes it also possible to compute the Hausdorff dimension of the sets

$$
\bigcap_{m=1}^{M}\left\{x \in X_{1}: \lim _{n \rightarrow \infty} \frac{\log \mu\left(R_{n}\left(\lambda_{1}^{(m)}, \ldots, \lambda_{k}^{(m)}, x\right)\right)}{-n}=\beta_{m}\right\},
$$

where $\beta \in \mathbb{R}_{+}^{M}$ and each $\left(\lambda_{i}^{(m)}\right)_{1 \leq i \leq m}$ satisfies $0 \leq \lambda_{1}^{(m)} \leq \cdots \leq \lambda_{k}^{(m)}$.

Remark 6.4. Let $\Phi \in \mathcal{C}_{a s a}\left(X_{1}, T_{1}\right)$. We say that a fully supported Borel probability measure $\mu$ (not necessarily to be shift invariant) on $X_{1}$ is an a-weighted weak Gibbs measure associated with $\Phi$ if

$$
\mu(I) \approx_{n} \exp \left(\frac{-n P}{A_{k}}\right) \phi_{n}^{\mathbf{a}}(I), \quad I \in \mathcal{A}^{n},
$$

where $P=P^{\mathbf{a}}\left(T_{1}, \Phi\right), A_{k}=a_{1}+\cdots+a_{k}, \Phi^{\mathbf{a}}=\left(\log \phi_{n}^{\mathbf{a}}\right) \in \mathcal{C}_{a s a}\left(X_{1}, T_{1}\right)$ is defined as in (3.4), and $\approx_{n}$ means that there exists a sequence of positive numbers $\left(\kappa_{n}\right)_{n=1}^{\infty}$ with $\lim _{n \rightarrow \infty}(1 / n) \log \kappa_{n}=0$, such that the ratio between the left and right hand sides of $\approx_{n}$ lies in $\left(\kappa_{n}^{-1}, \kappa_{n}\right)$.

This notion reduces back to classical weak Gibbs measures when $\mathbf{a}=(1,0 \ldots, 0)$ and $\Phi$ is the sequence of Birkhoff sums associated with a continuous potential over $X_{1}[48,29]$. It turns out that such a measure always exists for each $\Phi \in \mathcal{C}_{a s a}\left(X_{1}, T_{1}\right)$, and Theorem 6.3 can be extended to weak a-weighted equilibrium states (for details, see an earlier version [1] of this paper).

Appendix A. Main notation and conventions. For the reader's convenience, we summarize in Table A.1 the main notation and typographical conventions used in this paper. 
TABLE A.1

Main notation and conventions

\begin{tabular}{|c|c|}
\hline$\Phi=\left(\log \phi_{n}\right)_{n=1}^{\infty}$ & (Asymptotically sub-additive) potential (Section 1 ) \\
\hline $\mathcal{M}(X, T)$ & Set of $T$-invariant Borel probability measures on $X$ \\
\hline$h_{\mu}(T)$ & Measure-theoretic entropy of $T$ with respect to $\mu$ (Section 1 ) \\
\hline$\Phi_{*}(\mu)$ & $\lim _{n \rightarrow \infty} \frac{1}{n} \int \log \phi_{n}(x) d \mu(x)$ \\
\hline$P(T, \Phi, K)$ & $(\text { cf. }(2.2))^{\circ}$ \\
\hline$P(T, \Phi)$ & Topological pressure of $\Phi$ (Section 2.2) \\
\hline$h_{\mu}^{\mathbf{a}}\left(T_{1}\right)$ & $\begin{array}{l}\text { a-weighted measure-theoretic entropy of } T_{1} \text { with respect to } \mu \text { (Section } \\
\text { 1) }\end{array}$ \\
\hline$P^{\mathbf{a}}\left(T_{1}, \Phi\right)$ & a-weighted topological pressure of $\Phi($ Section 1$)$ \\
\hline $\mathcal{C}_{s}(X, T)$ & Collection of sub-additive potentials on $X$ (Section 1$)$ \\
\hline $\mathcal{C}_{a s s}(X, T)$ & Collection of asymptotically sub-additive potentials on $X$ (Section 1$)$ \\
\hline $\mathcal{C}_{a s a}(X, T)$ & Collection of asymptotically additive potentials on $X$ (Section 1$)$ \\
\hline $\mathcal{C}_{a a}(X, T)$ & Collection of almost additive potentials on $X$ (Section 1$)$ \\
\hline$C(X)$ & Collection of real continuous functions on $X$ \\
\hline$G_{\mu}(X, T)$ & Set of generic points of $\mu \in \mathcal{M}(X, T)$ (cf. (1.3)) \\
\hline$\Phi=\left(\Phi_{1}\right.$, & A family of asymptotically sub-additive potentials \\
\hline$\Phi_{*}(\mu)$ & $\left(\left(\Phi_{1}\right)_{*}(\mu), \ldots,\left(\Phi_{k}\right)_{*}(\mu)\right)$ \\
\hline$L_{\Phi}$ & $\left\{\boldsymbol{\Phi}_{*}(\mu): \mu \in \mathcal{M}\left(X_{1}, T_{1}\right)\right\}$ \\
\hline$\lambda_{\Phi}(x)$ & Lyapunov exponent of $\Phi$ at $x$ (Section 2.2) \\
\hline$E_{\left\{\boldsymbol{\Phi}_{j}\right\}_{j=1}^{r}, \mathbf{c}}(\alpha)$ & non-homogeneous $\alpha$-level set of $\left\{\boldsymbol{\Phi}_{j}\right\}_{j=1}^{r}$ (cf. Section 1) \\
\hline $\begin{array}{l}a_{n} \approx b_{n} \\
a_{n} \approx_{n} b_{n}\end{array}$ & $\begin{array}{l}(1 / c) b_{n} \leq a_{n} \leq c b_{n} \text { for a constant } c>0 \\
\left(1 / \kappa_{n}\right) b_{n} \leq a_{n} \leq \kappa_{n} b_{n} \text { for a sequence of positive numbers }\left(\kappa_{n}\right) \text { with } \\
\lim _{n \rightarrow \infty}(1 / n) \log \kappa_{n}=0\end{array}$ \\
\hline$\Phi^{\mathbf{a}}$ & (cf. $(3.4))$ \\
\hline$d_{\mathbf{a}}$ & "self-affine" metric (cf. (1.1)). \\
\hline
\end{tabular}

Acknowledgements. Both authors were partially supported by the France/Hong Kong joint research scheme PROCORE (projects 20650VJ, FHK08/08T). Feng was also partially supported by the RGC grant and the Focused Investments Scheme B in CUHK.

\section{REFERENCES}

[1] J. BARRAL AND D. J. Feng, Weighted thermodynamic formalism and applications, arXiv:0909.4247v1.

[2] J. Barral and M. Mensi, Gibbs measures on self-affine Sierpinski carpets and their singularity spectrum, Ergod. Th. \& Dynam. Sys., 27:5 (2007), pp. 1419-1443.

[3] J. Barral And M. Mensi, Multifractal analysis of Birkhoff averages on "self-affine" symbolic spaces, Nonlinearity, 21:10 (2008), pp. 2409-2425.

[4] L. BARREIRA, A non-additive thermodynamic formalism and applications to dimension theory of hyperbolic dynamical systems, Ergodic Theory Dynam. Systems, 16:5 (1996), pp. 871927.

[5] L. BARreira, Nonadditive thermodynamic formalism: equilibrium and Gibbs measures, Discrete Contin. Dyn. Syst., 16 (2006), pp. 279-305.

[6] L. Barreira, Dimension and recurrence in hyperbolic dynamics, Progress in Mathematics, 272. Birkhäuser Verlag, Basel, 2008.

[7] T. Bedford, Crinkly curves, Markov partitions and box dimension in self-similar sets, Ph.D. Thesis, University of Warwick, 1984.

[8] P. Billingsley, Ergodic theory and information, Wiley, New York, 1965.

[9] R. Bowen, Topological entropy for noncompact sets, Trans. Amer. Math. Soc., 184 (1973), pp. $125-136$.

[10] R. Bowen, Equilibrium states and the ergodic theory of Anosov diffeomorphisms, Lecture notes in Math., No. 470, Springer-Verlag, 1975.

[11] H. CAJAR, Billingsley dimension in probability spaces, Lecture Notes in Mathematics, 892, Springer-Verlag, Berlin-New York, 1981. 
[12] Y. L. CaO, D. J. Feng And W. HuAng, The thermodynamic formalism for sub-additive potentials, Discrete Contin. Dyn. Syst., 20 (2008), pp. 639-657.

[13] J.-R. Chazottes and E. UGalde, Projection of Markov measures may be Gibbsian, J. Statist. Phys., 111 (2003), pp. 1245-1272.

[14] J.-R. Chazottes And E. UGalde, On the preservation of Gibbsianness under symbol amalgamation, arXiv:0907.0528

[15] P. Collet, J. L. Lebowitz and A. Porzio, The dimension spectrum of some dynamical systems, J. Statist. Phys., 47 (1987), pp. 609-644.

[16] T. Downarowicz And J. SERAfin, Fiber entropy and conditional variational principles in compact non-metrizable spaces, Fund. Math., 172:3 (2002), pp. 217-247.

[17] K. J. FALCOner, A subadditive thermodynamic formalism for mixing repellers, J. Phys. A, 21:14 (1988), pp. L737-L742.

[18] A. H. FAN, Sur la dimension des mesures, Studia Mathematica, 111 (1994), pp. 1-17.

[19] A. H. Fan, D. J. Feng And J. Wu, Recurrence, dimension and entropy, J. London Math. Soc. (2), 64:1 (2001), pp. 229-244.

[20] A. H. FAn, L. LiAO AND J. Peyrière, Generic points in systems of specification and Banach valued Birkhoff ergodic average, Discrete Contin. Dyn. Syst., 21 (2008), pp. 1103-1128.

[21] D. J. FEnG, The variational principle for products of non-negative matrices, Nonlinearity, 17 (2004), pp. 447-457.

[22] D. J. FEnG, Equilibrium states for factor maps between subshifts, Adv. Math., 226 (2011), pp. 2470-2502.

[23] D. J. FENG AND W. HuANG, Lyapunov spectrum of asymptotically sub-additive potentials, Commun. Math. Phys., 297 (2010), pp. 1-43.

[24] D. J. FENG AND K. S. LAU, The pressure function for products of non-negative matrices, Math. Res. Lett., 9 (2002), pp. 363-378.

[25] D. Gatzouras And Y. Peres, Invariant measures of full dimension for some expanding maps, Ergod. Th. \& Dynam. Sys., 17:1 (1997), pp. 147-167.

[26] J. P. Hiriart-Urruty and C. Lemaréchal, Fundamentals of convex analysis. SpringerVerlag, Berlin, 2001.

[27] A. K̈̈ЕNM̈̈KI, On natural invariant measures on generalised iterated function systems, Ann. Acad. Sci. Fenn. Math., 29 (2004), pp. 419-458.

[28] R. Kenyon and Y. Peres, Measures of full dimension on affine-invariant sets, Ergod. Th. \& Dynam. Sys., 16 (1996), pp. 307-323.

[29] M. KesseböHMeR, Large deviation for weak Gibbs measures and multifractal spectra, Nonlinearity, 14 (2001), pp. 395-409.

[30] M. Kesseböhmer and B. O. Stratmann, A multifractal formalism for growth rates and applications to geometrically finite Kleinian groups, Ergodic Theory Dynam. Systems, 24 (2004), pp. 141-170.

[31] J. F. KIng, The singularity spectrum for general Sierpinski carpets, Adv. Math., 116 (1995), pp. $1-8$.

[32] F. LEDRAPPIER AND P. WALters, A relativised variational principle for continuous transformations, J. Lond. Math. Soc., 16 (1977), pp. 568-576.

[33] D. Lind And B. Marcus, An Introduction to Symbolic Dynamics and Coding, Cambridge University Press, Cambridge, 1995.

[34] P. Mattila, Geometry of sets and measures in Euclidean spaces. Fractals and rectifiability, Cambridge University Press, 1995.

[35] C. McMullen, The Hausdorff dimension of general Sierpinsky carpets, Nagoya Math. J. 96 (1984), 1-9.

[36] A. Mummert, The thermodynamic formalism for almost-additive sequences, Discrete Contin. Dyn. Syst., 16 (2006), pp. 435-454.

[37] L. OLsen, Self-affine multifractal Sierpinski sponges in $\mathbb{R}^{d}$, Pacific J. Math., 183 (1998), pp. $143-199$.

[38] YA. B. PEsin, Dimension theory in dynamical systems. Contemporary views and applications, University of Chicago Press, 1997.

[39] C. E. Pfister And W. G. Sullivan, On the topological entropy of saturated sets, Ergod. Th. \& Dynam. Sys., 27 (2007), pp. 929-956.

[40] D. A. RAnd, The singularity spectrum $f(\alpha)$ for cookie-cutters, Ergod. Th. \& Dynam. Sys., 9 (1989), pp. 527-541.

[41] H. WJ. REEVE, The packing spectrum for Birkhoff averages on a self-affine repeller, arXiv:math/1008.0301.

[42] R. T. Rockafellar, Convex analysis, Princeton Mathematical Series, No. 28, Princeton University Press, Princeton, N. J. 1970. 
[43] D. RuelLe, Thermodynamic formalism. The mathematical structures of classical equilibrium statistical mechanics, Encyclopedia of Mathematics and its Applications, 5. AddisonWesley Publishing Co., Reading, Mass., 1978.

[44] P. Walters, An introduction to ergodic theory, Springer-Verlag, 1982.

[45] Y. ZhaO AND Y. L. CAO, On the topological pressure of random bundle transformations in sub-additive case, J. Math. Anal. Appl., 342 (2008), pp. 715-725.

[46] Y. YAYAma, Existence of a measurable saturated compensation function between subshifts and its applications, Ergodic Theory Dynam. Systems, 31 (2011), pp. 1563-1589.

[47] Y. YAYAMA, Applications of a relative variational principle to dimensions of nonconformal expanding maps, Stoch. Dyn., 11 (2011), pp. 643-679.

[48] M. YURI, Zeta functions for certain non-hyperbolic systems and topological Markov approximations, Ergod. Th. \& Dynam. Sys., 17 (1997), pp. 997-1000. 